\title{
Twist-3 contributions to wide-angle photoproduction of pions
}

\author{
P. Kroll \\ Fachbereich Physik, Universität Wuppertal, D-42097 Wuppertal, Germany \\ K. Passek-Kumerički \\ Theoretical Physics Division, Rudjer Bošković Institute, HR-10002 Zagreb, Croatia
}

(Received 26 February 2018; published 23 April 2018)

\begin{abstract}
We investigate wide-angle $\pi^{0}$ photoproduction within the handbag approach to twist-3 accuracy. In contrast to earlier work both the 2-particle as well as the 3-particle twist-3 contributions are taken into account. It is shown that both are needed for consistent results that respect gauge invariance and crossing properties. The numerical studies reveal the dominance of the twist-3 contribution. With it fair agreement with the recent CLAS measurement of the $\pi^{0}$ cross section is obtained. We briefly comment also on wide-angle photoproduction of other pseudoscalar mesons.
\end{abstract}

DOI: $10.1103 /$ PhysRevD.97.074023

\section{INTRODUCTION}

Since 1996 there have been a lot of activities on the field of hard exclusive processes in conjunction with handbag factorization. A vast amount of data on such processes has been accumulated from HERMES, COMPASS, BABAR, and BELLE and from experiments performed at Jefferson Lab and HERA. Many theoretical studies of these processes have been carried through within the framework of the handbag approach in which the process amplitudes factorize in hard, perturbatively calculable subprocesses and soft hadron matrix elements, parametrized as generalized parton distributions (GPDs).

Of particular importance for the present work is wideangle Compton scattering (WACS). There are reasonable arguments $[1,2]$ that for large Mandelstam variables, $s,-t$, and $-u$, the Compton amplitudes can be represented as a product of amplitudes for the subprocess, Compton scattering off quarks, and form factors that represent $1 / x$ moments of GPDs. Since the GPDs in question, namely $H, E$, and $\tilde{H}$, are known from an analysis of the form factors of the nucleon [3] one can compute the Compton form factors and subsequently the Compton cross section as well as other observables for this process. The results of this parameter-free prediction [3] agrees quite well with experiment [4] given that the Mandelstam variables achieved in current experiments are not large as compared to a typical hadronic scale of order $1 \mathrm{GeV}^{2}$. An analogous calculation of wide-angle photoproduction

Published by the American Physical Society under the terms of the Creative Commons Attribution 4.0 International license. Further distribution of this work must maintain attribution to the author(s) and the published article's title, journal citation, and DOI. Funded by SCOAP ${ }^{3}$. of mesons however fails [5]: the cross sections are underestimated by about 2 orders of magnitude. An attempt to improve this result has been presented in [6]: under the assumption of a vanishing contribution from the $q \bar{q} g$ Fock component of the meson (frequently termed the Wandzura-Wilczek approximation) the 2-particle twist-3 meson distribution amplitudes (DAs) have been taken into account along with the helicity-flip or transversity GPDs $[7,8]$. The analysis, however, revealed that the corresponding 2-particle twist-3 contribution is zero. Thus, this attempt turned out to be unsuccessful.

A HERMES measurement [9] of the asymmetry in electroproduction of positively charged pions, obtained with a transversely polarized target, indicated a strong contribution from transversely polarized virtual photons which in the generalized Bjorken regime of large photon virtuality, $Q^{2}$, and large photon-proton center-of-mass energy but fixed Bjorken- $x$ and $-t \ll Q^{2}$ is in principle suppressed by $1 / Q^{2}$ in the cross section as compared to the asymptotically leading contribution from longitudinally polarized photons [10]. In [11,12] it has been shown that the HERMES result on the asymmetry can be understood by just the same dynamical mechanism, namely the combination of transversity GPDs and twist-3 pion DAs, that failed in wide-angle photoproduction as we mentioned above. We stress that in pion electroproduction the mechanism in question is probed at large $Q^{2}$ but $t \rightarrow 0$ in contrast to photoproduction where $-t$ (and $-u$ ) are large but $Q^{2} \rightarrow 0$. The twist-3 contribution is large in the case of pions because it is proportional to a mass parameter, $\mu_{\pi}$, which is related to the chiral condensate

$$
\mu_{\pi}=\frac{m_{\pi}^{2}}{m_{u}+m_{d}}
$$


by means of the divergence of the axial vector current. Here, $m_{i}$ are current quark masses and $m_{\pi}$ denotes the mass of the pion. This parameter is large, about $2 \mathrm{GeV}$ at the scale $2 \mathrm{GeV}$. The transverse cross section for pion electroproduction is parametrically suppressed by $\mu_{\pi}^{2} / Q^{2}$ as compared to the longitudinal cross section. For the accessible range of $Q^{2}$ in current experiments the suppression factor is of order unity. Predictions for the $\pi^{0}$ electroproduction cross sections given in [12] (see also [13]) revealed a transverse cross section that is much larger than the longitudinal one. This prediction has been confirmed by a recent measurement of the separated $\pi^{0}$ cross sections performed by the Jefferson Lab Hall A Collaboration [14]. The longitudinal cross section is found to be compatible with zero within the experimental errors. A preliminary COMPASS result [15] for the unseparated cross section at a much larger center-of-mass energy but approximately the same $Q^{2}$ is, in tendency, in agreement with the Hall $\mathrm{A}$ findings. Thus, the same situation appears in both hard $\pi^{0}$ electroproduction and wide-angle $\pi^{0}$ photoproduction-a leading-twist analysis fails badly in comparison with experiment at presently available hard scales.

In view of these experimental and theoretical results on hard exclusive pion electroproduction a resumption of the investigation on the wide-angle meson photoproduction seems to be appropriate, and this is the purpose of the present work. It differs from the earlier work $[5,6]$ by the inclusion of the full, genuine twist-3 contribution, i.e., its 2-particle as well as its 3-particle parts. Both parts are related to each other by the equation of motion [16] and both are required in order to accomplish gauge invariance and crossing properties. In Sec. II we recapitulate the handbag approach to photoproduction of uncharged pions to twist-3 accuracy. In the next section, Sec. III, we discuss the large $-t$ behavior of the relevant helicity flip and nonflip GPDs and the corresponding form factors. The subprocess amplitudes to twist-3 accuracy are discussed in Sec. IV and, in Sec. V, results for the cross section and spin-dependent observables for photoproduction of the $\pi^{0}$ are presented. There are also comments on photoproduction of other mesons. The paper is finished with the usual summary. In Appendix A the 2- and 3-particle twist-3 DAs are discussed in some detail. The separate 2- and 3-particle twist-3 subprocess amplitudes are presented in Appendix B.

\section{THE HANDBAG MECHANISM}

The handbag mechanism for wide-angle photoproduction of uncharged pions, $\gamma p \rightarrow \pi^{0} p$, where $p$ denotes a proton, has been developed in $[5,6]$. For a better comprehension of the present work we are going to recapitulate the main results and arguments for factorization of the photoproduction amplitude in hard subprocesses and soft form factors.

Prerequisite is that the Mandelstam variables $s,-t$, and $-u$ are much larger than $\Lambda^{2}$ where $\Lambda$ is a typical hadronic scale of order $1 \mathrm{GeV}$. It is advantageous to work in a symmetrical frame which is a center-of-mass frame (c.m.s.) rotated in such a way that the momenta of the ingoing $(p)$ and outgoing $\left(p^{\prime}\right)$ nucleons have the same light-cone plus components

$$
\begin{aligned}
p & =\left[p^{+}, \frac{m^{2}-t / 4}{2 p^{+}},-\frac{1}{2} \boldsymbol{\Delta}_{\perp}\right], \\
p^{\prime} & =\left[p^{+}, \frac{m^{2}-t / 4}{2 p^{+}}, \frac{1}{2} \boldsymbol{\Delta}_{\perp}\right],
\end{aligned}
$$

where $m$ is the mass of the proton. In this frame the skewness, defined by

$$
\xi=\frac{\left(p-p^{\prime}\right)^{+}}{\left(p+p^{\prime}\right)^{+}}
$$

is zero. We assume restricted parton virtualities $k_{i}^{2}<\Lambda^{2}$ and intrinsic transverse parton momenta, $k_{\perp i}$, defined with respect to their parent hadron's momentum, which satisfy the condition $k_{\perp i}^{2} / x_{i}<\Lambda^{2}$. Here, $x_{i}$ denotes the momentum fraction that parton $i$ carries. On these premises one can show [5] that the subprocess Mandelstam variables $\hat{s}$ and $\hat{u}$ coincide with the ones for the full process, photoproduction of pions, up to corrections ${ }^{1}$ of order $\Lambda^{2} / s$,

$$
\begin{aligned}
& \hat{t}=t, \quad \hat{s}=\left(k_{j}+q\right)^{2} \simeq(p+q)^{2}=s, \\
& \hat{u}=\left(k_{j}^{\prime}-q\right)^{2} \simeq\left(p^{\prime}-q\right)^{2}=u,
\end{aligned}
$$

where $k_{j}$ and $k_{j}^{\prime}=k_{j}+q-q^{\prime}$ denote the momenta of the active partons, i.e., the in and out partons to which the photon couples; $q$ and $q^{\prime}$ are the momenta of the photon and meson, respectively. Thus, the active partons are approximately on shell, move collinear with their parent hadrons, and carry a momentum fraction close to unity, $x_{j}, x_{j}^{\prime} \simeq 1$. As in deeply virtual exclusive scattering, the physical situation is that of a hard parton-level subprocess, $\gamma q_{a} \rightarrow \pi^{0} q_{a}$, and a soft emission and reabsorption of quarks from the proton. Up to corrections of order $\Lambda / \sqrt{-t}$ the light-cone helicity amplitudes for wide-angle photoproduction are then given by a product of subprocess amplitudes, $\mathcal{H}$, and form factors

$$
\begin{aligned}
\mathcal{M}_{0+, \mu+}= & \frac{e_{0}}{2} \sum_{\lambda}\left[\mathcal{H}_{0 \lambda, \mu \lambda}\left(R_{V}^{\pi^{0}}(t)+2 \lambda R_{A}^{\pi^{0}}(t)\right)\right. \\
& \left.-2 \lambda \frac{\sqrt{-t}}{2 m} \mathcal{H}_{0-\lambda, \mu \lambda} \bar{S}_{T}^{\pi^{0}}(t)\right], \\
\mathcal{M}_{0-, \mu+}= & \frac{e_{0}}{2} \sum_{\lambda}\left[\frac{\sqrt{-t}}{2 m} \mathcal{H}_{0 \lambda, \mu \lambda} R_{T}^{\pi^{0}}(t)\right. \\
& \left.-2 \lambda \frac{t}{2 m^{2}} \mathcal{H}_{0-\lambda, \mu \lambda} S_{S}^{\pi^{0}}(t)\right]+e_{0} \mathcal{H}_{0-, \mu+} S_{T}^{\pi^{0}}(t),
\end{aligned}
$$

${ }^{1}$ Possible corrections due to the proton mass have been discussed in [17]. 
where $\mu$ denotes the helicity of the photon, $\lambda$ the helicity of the active quark, and $e_{0}$ the positron charge. Note that for the sake of legibility helicities are labeled by their signs only. The amplitudes for helicity configurations other than quoted in (5) follow from parity invariance:

$$
\mathcal{M}_{0-\nu^{\prime},-\mu-\nu}=(-1)^{\nu-\nu^{\prime}} \mathcal{M}_{0 \nu^{\prime}, \mu \nu} .
$$

An analogous relation holds for the subprocess amplitudes $\mathcal{H}$. The soft form factors, $R_{i}^{\pi^{0}}$ and $S_{i}^{\pi^{0}}$, are specific to photoproduction of uncharged pions. They represent $1 / x$-moments of GPDs at zero skewness, where $x=\left(k_{j}+\right.$ $\left.k_{j}^{\prime}\right)^{+} /\left(p+p^{\prime}\right)^{+}$is the average momentum fraction the two active quarks carry. The form factors parametrize the soft physics that controls the emission from and reabsorption of a quark by the proton. They will be discussed in some detail in the next section. The representation (5), which requires the dominance of the plus components of the proton matrix elements, is a nontrivial feature given that, in contrast to deep inelastic lepton-nucleon and deep virtual exclusive processes, not only the plus components of the proton momenta but also their minus and transverse components are large in this case [2]. The generalization of (5) to photoproduction of other pseudoscalar mesons is straightforward [6].

\section{GPDs AND FORM FACTORS AT LARGE-t}

The form factors for an active quark of flavor $a$ are defined by $[5,6]$

$$
\begin{aligned}
R_{V}^{a}(t) & =\int_{-1}^{1} \frac{d x}{x} \operatorname{sign}(x) H^{a}(x, t), \\
S_{T}^{a}(t) & =\int_{-1}^{1} \frac{d x}{x} \operatorname{sign}(x) H_{T}^{a}(x, t), \\
R_{A}^{a}(t) & =\int_{-1}^{1} \frac{d x}{x} \tilde{H}^{a}(x, t), \\
S_{S}^{a}(t) & =\int_{-1}^{1} \frac{d x}{x} \operatorname{sign}(x) \tilde{H}_{T}^{a}(x, t), \\
R_{T}^{a}(t) & =\int_{-1}^{1} \frac{d x}{x} \operatorname{sign}(x) E^{a}(x, t), \\
S_{V}^{a}(t) & =\int_{-1}^{1} \frac{d x}{x} \operatorname{sign}(x) E_{T}^{a}(x, t) .
\end{aligned}
$$

It is also convenient to introduce the combination

$$
\bar{S}_{T}^{a}(t)=2 S_{S}^{a}(t)+S_{V}^{a}(t)
$$

associated with the GPD $\bar{E}_{T}=2 \tilde{H}_{T}+E_{T}$. The functions $H^{a}, \tilde{H}^{a}$, and $E^{a}$ are the familiar helicity nonflip GPDs at zero skewness, whereas $H_{T}^{a}, \tilde{H}_{T}^{a}$, and $E_{T}^{a}$ denote the helicity flip or transversity GPDs. The skewness variable is omitted in the GPDs for convenience. The GPDs $\tilde{E}^{a}$ and $\tilde{E}_{T}^{a}$ and their associated form factors decouple in the symmetrical frame. Note that $x$ runs from -1 to +1 . As usual a parton with a negative momentum fraction is reinterpreted as an antiproton with a positive momentum fraction. One has

$$
K^{\bar{a}}(x, t)=-K^{a}(-x, t) \quad(x>0)
$$

for all GPDs, $K$, except for $\tilde{H}$ for which the relation

$$
\tilde{H}^{\bar{a}}(x, t)=\tilde{H}^{a}(-x, t) \quad(x>0)
$$

holds. Thus, the flavor form factors in (7) can also be written as $\left(F_{i}=R_{V}, R_{A}, \ldots, S_{V}\right)$

$$
F_{i}^{a}(t)=\int_{0}^{1} \frac{d x}{x}\left(K_{i}^{a}(x, t)-K_{i}^{\bar{a}}(x, t)\right) .
$$

One notices that quarks and antiquarks contribute with opposite signs to photoproduction of pseudoscalar mesons; i.e., only valence quarks contribute. This is to be contrasted with Compton scattering [2] or photoproduction of vector mesons [5] where they contribute with the same sign. This feature reflects the charge-conjugation properties of the GPDs.

The flavor form factors are to be combined in form factors specific to a given process. Thus, for the process on which we focus our interest, $\pi^{0}$ photoproduction off protons, the relevant combination of the flavor form factors is

$$
F_{i}^{\pi^{0}}(t)=\frac{1}{\sqrt{2}}\left[e_{u} F_{i}^{u}(t)-e_{d} F_{i}^{d}(t)\right],
$$

where $e_{a}$ is the charge of a quark of flavor $a$ in units of the positron charge, $e_{0}$.

In [3] the GPDs $H$ and $E$ for valence quarks have been extracted from the data on the magnetic form factors of the proton and the neutron and from the ratio of electric and magnetic form factors exploiting the sum rules for the form factors with the help of a parametrization of the zero skewness GPDs

$$
K_{i}^{a}=k_{i}^{a}(x) \exp \left[t f_{i}^{a}(x)\right] .
$$

In $[3,18]$ it is advocated for the following parametrization of the profile function:

$$
f_{i}^{a}(x)=\left(B_{i}^{a}-\alpha_{i}^{\prime a} \ln x\right)(1-x)^{3}+A_{i}^{a} x(1-x)^{2},
$$

with $A_{i}, B_{i}$, and $\alpha_{i}$ being the parameters discussed below. The forward limit of the GPD $H^{a}$ is given by the flavor-a parton density, $q^{a}(x)$. On the other hand, the forward limit of $E^{a}$ is not accessible in deep-inelastic scattering and is, therefore, to be determined in the form factor analysis, too. For the parametrization (13) and (14) there is a strong $x-t$ correlation in the GPD as has been discussed in $[3,18]$. The GPDs at small $x$ control the behavior of the associated 
flavor form factors at small $-t$, whereas large $x$ determine their large $-t$ behavior which is required for wide-angle photoproduction of mesons. As is obvious from (14) at small $x$ the first term of the profile function dominates while at large $x$ its second term is important. In analyses of deeply virtual exclusive processes, as electroproduction of photons or meson, for which only data at rather small $-t$ are available, the so-called Regge-like profile function is frequently used. This profile function is just the first term of (14) with the factor $(1-x)^{3}$ being dropped. Clearly, in view of the $x-t$ correlation, we can only learn about the GPDs at small $x$ from data on deeply virtual exclusive processes; an extrapolation to large $x$ and large $-t$ is dangerous and may lead to misleading results. ${ }^{2}$ The parameter $A$ of the second term in (14) cannot be fixed from small $-t$ data. Information on the large $-t$ (large $x$ ) behavior of the GPDs is for instance obtained from the electromagnetic form factors of the nucleon.

After these preliminaries we move on to the discussion of the actual choice of the form factors: $R_{V}^{\pi^{0}}$ and $R_{T}^{\pi^{0}}$ are evaluated from the GPDs derived in [3]. The GPD $\tilde{H}$ is known only for $-t$ less than about $3 \mathrm{GeV}^{2}[3]$ from the data on the axial form factor [19]. Data at larger $-t$ are to be expected from the upcoming Fermilab MINERvA experiment. From data on the helicity correlations $A_{\mathrm{LL}}$ and/or $K_{\mathrm{LL}}$ in wide-angle Compton scattering we may also learn about the large $-t$ behavior of the GPD $\tilde{H}$ [20]. Measurements of these helicity correlations are planned at Jefferson Lab. On the basis of the parametrization (13) and (14) (with the unpolarized parton densities replaced by the polarized ones) and the results on $\tilde{H}$ given in [3] several examples of the large $-t$ behavior of $\tilde{H}$ are discussed in [20]. For the numerical estimates of observables for wide-angle photoproduction of pseudoscalar mesons to be presented in Sec. V below we will use example 1 quoted in [20].

In $[11,12]$ hard pion electroproduction has been studied and the valence quark GPDs $H_{T}$ and $\bar{E}_{T}$ at small $-t$ extracted. These GPDs are also parametrized as in (13) and (14). Their forward limits read

$$
\begin{aligned}
h_{T}^{a} & =N_{H}^{a} x^{1 / 2}(1-x)\left[q^{a}(x)+\Delta q^{a}(x)\right], \\
\bar{e}_{T}^{a} & =N_{E}^{a} x^{-\alpha_{e_{T}}^{a}}(1-x)^{\beta_{e_{T}}^{a}} .
\end{aligned}
$$

The particular parametrization of the forward limit of $H_{T}$ guarantees that the Soffer bound is respected. For the numerical studies the parton densities are taken from [21,22]. The parameters of the GPDs $H_{T}$ and $\bar{E}_{T}$ are quoted in Table I. In addition there are the parameters of the forward limit of $\bar{e}_{T}$,

$$
\alpha_{e_{T}}^{u}=\alpha_{e_{T}}^{u}=0.3, \quad \beta_{e_{T}}^{u}=4, \quad \beta_{e_{T}}^{d}=5,
$$

which are also taken from [12].

\footnotetext{
${ }^{2}$ As shown in [18] the Regge-like profile function leads to an infinitely large distance between the active quark and the cluster of spectators; i.e., it leads to a violation of confinement.
}

TABLE I. Parameters of the GPDs $H_{T}$ and $\bar{E}_{T}$ taken from [12].

\begin{tabular}{llccc}
\hline \hline & $H_{T}^{u}$ & $H_{T}^{d}$ & $\bar{E}_{T}^{u}$ & $\bar{E}_{T}^{d}$ \\
\hline$N$ & 0.78 & -1.01 & 4.83 & 3.57 \\
$\alpha^{\prime}\left[\mathrm{GeV}^{-2}\right]$ & 0.45 & 0.45 & 0.45 & 0.45 \\
$B\left[\mathrm{GeV}^{-2}\right]$ & 0 & 0 & 0.50 & 0.50 \\
\hline \hline
\end{tabular}

As mentioned before and shown in Fig. 1 the Reggelike profile function leads to form factors that rapidly drop with $-t$. Clearly, also for wide-angle photoproduction of pseudoscalar mesons the second term of the profile function (14) is required. In the absence of any information on the parameter $A$ we tentatively choose for it the value $0.5 \mathrm{GeV}^{-2}$ in all cases. Fortunately, the dependence of the form factors $S_{i}$ on that parameter is rather mild in the range of $t$ relevant to current photoproduction experiments. This is demonstrated by the band for $\bar{S}_{T}^{\pi^{0}}$ evaluated from $A=0.3 \mathrm{GeV}^{-2}$ and $0.7 \mathrm{GeV}^{-2}$. The form factor $\bar{S}_{T}^{\pi^{0}}$ is rather large since $\bar{E}_{T}^{u}$ and $\bar{E}_{T}^{d}$ have the same sign and about the same normalization, a fact that is supported by results from lattice QCD [23]. This feature of $\bar{E}_{T}$ is also responsible for the dominance of this GPD in electroproduction of $\pi^{0}$.

With the help of the saddle point method [18] one can show that the moments of the GPDs, parametrized by (13) and (14), behave power-law-like:

$$
F_{i} \sim 1 /(-t)^{d_{i}}
$$

The power $d_{i}$ is determined by the power $\beta_{i}$ of the factor $1-x$ that characterizes the behavior of the forward limits of the GPDs for $x \rightarrow 1$,

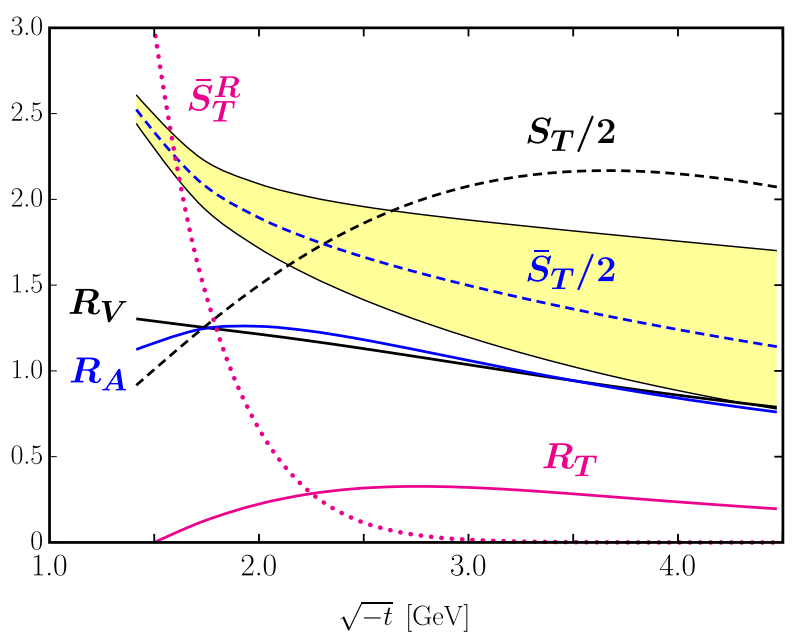

FIG. 1. The form factors for $\pi^{0}$ photoproduction scaled by $t^{2}$. The dimension is $\mathrm{GeV}^{4}$. For the transversity form factor the value of the parameter $A$ is $0.5 \mathrm{GeV}^{-2}$. The upper (lower) edge of the band for $\bar{S}_{T}$ is evaluated from $A=0.3(0.7) \mathrm{GeV}^{-2}$. 
TABLE II. The powers $d_{i}$ for the various form factors contributing to the wide-angle photoproduction of pseudoscalar mesons.

\begin{tabular}{llllll}
\hline \hline & $R_{V}$ & $R_{A}$ & $R_{T}$ & $S_{T}$ & $\bar{S}_{T}$ \\
\hline$u$ & 2.25 & 2.22 & 2.83 & 2.5 & 2.5 \\
$d$ & 3.0 & 2.61 & 3.12 & 3.5 & 3.0 \\
\hline \hline
\end{tabular}

$$
d_{i}=\left(1+\beta_{i}\right) / 2 .
$$

We stress that the power $\beta_{i}$ is fixed in a region of $x$ less than about 0.8 . For larger $x$ there is no experimental information on the forward limits available at present. Therefore, the powers $\beta_{i}$ are to be considered rather as effective powers which are likely subject to change as soon as data at larger $x$ become available. The current powers $d_{i}$ are listed in Table II.

At present there is no information available on the GPD $\tilde{H}_{T}$ and its associated form factor $S_{S}$. It has been neglected in the analysis of electroproduction of pseudoscalar mesons because its contribution is suppressed by a factor $t /\left(2 m^{2}\right)$ [see also (5)]. However, this argument no longer holds for wide-angle meson photoproduction since $-t$ is large. As an estimation of its significance we take $S_{S}^{\pi^{0}} \simeq$ $\bar{S}_{T}^{\pi^{0}} / 2\left[S_{V}^{\pi^{0}} \simeq 0\right.$; cf. (8)].

\section{THE SUBPROCESS AMPLITUDES}

We calculate the amplitudes for the subprocess $\gamma q_{a} \rightarrow$ $\pi^{0} q_{a}$ to twist- 3 accuracy. In the definitions of the various vacuum-meson matrix elements as frequently done in QCD calculations of exclusive processes, we are using the lightcone (axial) gauge. All possible Wilson lines become unity in that gauge. Our calculation method is similar to the lightcone collinear factorization approach discussed in detail in [24,25] for the case of electroproduction of transversely polarized vector mesons.

Typical lowest-order Feynman graphs for the process of interest are depicted in Fig. 2. In particular the four graphs of type (a) are relevant for the 2-particle contributions. With the help of the $q \bar{q} \rightarrow \pi^{0}$ projector [26,27]

$$
\begin{aligned}
\mathcal{P}_{2, f g}= & \frac{f_{\pi}}{2 \sqrt{2 N_{C}}} \frac{\delta_{f g}}{\sqrt{N_{C}}}\left\{\frac{\gamma_{5}}{\sqrt{2}} q^{\prime} \phi_{\pi}(\tau)+\mu_{\pi} \frac{\gamma_{5}}{\sqrt{2}}\right. \\
& \times\left[\phi_{\pi p}(\tau)-\frac{i}{6} \sigma_{\mu \nu} \frac{q^{\prime \mu} k_{j}^{\prime \nu}}{q^{\prime} \cdot k_{j}^{\prime}} \phi_{\pi \sigma}^{\prime}(\tau)\right. \\
& \left.\left.+\frac{1}{6} \sigma_{\mu \nu} q^{\prime \mu} \phi_{\pi \sigma}(\tau) \frac{\partial}{\partial k_{\perp \nu}}\right]\right\}_{k_{\perp} \rightarrow 0}
\end{aligned}
$$

the subprocess amplitudes for the twist- 2 and for the 2-particle twist-3 contributions have already been calculated in [6]. The usual twist-2 pion DA is denoted by $\phi$ and $\phi_{p}, \phi_{\sigma}$ are the two 2-particle twist-3 DAs while $\phi_{\sigma}^{\prime}=d \phi_{\sigma} / d \tau$. Their definitions are given in Appendix A. In (19) $f_{\pi}$ is the familiar decay constant of the meson $\left(f_{\pi}=0.132 \mathrm{MeV}\right) ; \tau$ denotes the momentum fraction the quark entering the meson carries; $N_{C}$ is the number of colors; and $f$ and $g$ represent color labels of the quark and antiquark, respectively. The Dirac labels are omitted for convenience. In (19), $k_{\perp}$ denotes the intrinsic transverse momentum of the quark entering the meson, defined with respect to the meson's momentum, $q^{\prime}$. It is usually neglected in the collinear hard-scattering approach. The quark and antiquark momenta are thus given by

$$
k_{q}=\tau q^{\prime}+k_{\perp}, \quad k_{\bar{q}}=\bar{\tau} q^{\prime}-k_{\perp},
$$

where $\bar{\tau}=1-\tau$. After the derivative in (19) is performed, the collinear limit, $k_{\perp}=0$, is to be taken. Finally, the mass parameter $\mu_{\pi}$ is defined in Eq. (1). Taking from [28] the current-quark masses appearing in Eq. (1), one obtains $\mu_{\pi}\left(\mu_{0}^{2}\right)=2.6 \mathrm{GeV}$ at the scale $\mu_{0}^{2}=4 \mathrm{GeV}^{2}$. The uncertainty of $\mu_{\pi}$ is, however, large. ${ }^{3}$ The mass parameter evolves as

$$
\mu_{\pi}\left(\mu_{R}^{2}\right)=L^{-4 / \beta_{0}} \mu_{\pi}\left(\mu_{0}^{2}\right),
$$

where

$$
L=\frac{\alpha_{S}\left(\mu_{R}^{2}\right)}{\alpha_{S}\left(\mu_{0}^{2}\right)}=\frac{\ln \left(\mu_{0}^{2} / \Lambda_{\mathrm{QCD}}^{2}\right)}{\ln \left(\mu_{R}^{2} / \Lambda_{\mathrm{QCD}}^{2}\right)}
$$

and $\beta_{0}=\left(11 N_{C}-2 n_{f}\right) / 3$. We work with four flavors $\left(n_{f}=4\right)$ and adopt the value $\Lambda_{\mathrm{QCD}}=0.22 \mathrm{GeV}$. For the factorization and renormalization scale we choose $\mu_{F}^{2}=$ $\mu_{R}^{2}$ and

$$
\mu_{R}^{2}=\frac{\hat{t} \hat{u}}{\hat{s}},
$$

which takes care of the requirement that both $t$ and $u$ should be large.

The twist-2 contribution only affects the subprocess amplitude for quark helicity nonflip. At leading order (LO) of perturbative QCD it reads [6]

$$
\begin{aligned}
\mathcal{H}_{0 \lambda, \mu \lambda}^{\mathrm{twist}-2}= & 2 \pi \alpha_{s}\left(\mu_{R}^{2}\right) f_{\pi} \frac{C_{F}}{N_{C}} \frac{\sqrt{-\hat{t} / 2}}{\hat{u} \hat{s}} \\
& \times\langle 1 / \tau\rangle_{\pi}[(1+2 \lambda \mu) \hat{s}-(1-2 \lambda \mu) \hat{u}],
\end{aligned}
$$

where as usual $C_{F}=\left(N_{C}^{2}-1\right) /\left(2 N_{C}\right)$ is a color factor, while $\langle 1 / \tau\rangle$ is the $1 / \tau$ moment of twist- 2 pion DA. The symmetry of twist-2 pion DA under the replacement $\tau \leftrightarrow \bar{\tau}$ is in (24) already taken into account. For this DA we use the truncated Gegenbauer expansion

\footnotetext{
${ }^{3}$ For instance values of $1.8 \mathrm{GeV}$ and $1.9 \mathrm{GeV}$ at $\mu_{0}^{2}$ are quoted in [29,30], respectively.
} 

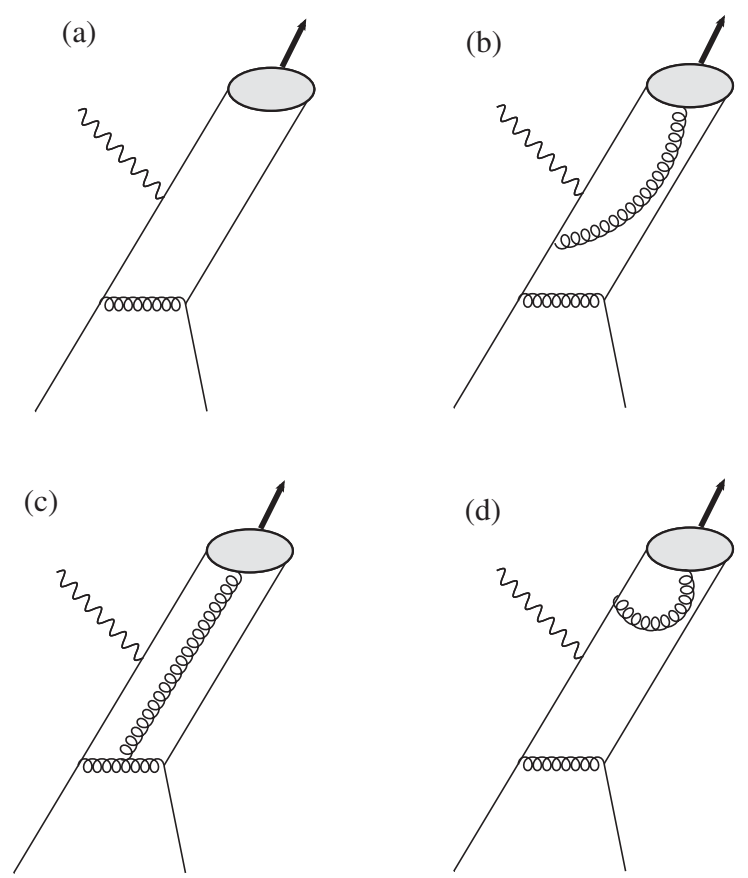

FIG. 2. Typical leading-order Feynman graphs for $\gamma q \rightarrow \pi^{0} q$. (a) For a 2-particle Fock component of a pseudoscalar meson. (b), (c) Contribution from the $q \bar{q} g$ Fock component without and with triple gluon coupling. (d) A soft contribution which is to be considered as part of the 3-particle DA.

$$
\phi_{\pi}\left(\tau, \mu_{R}^{2}\right)=6 \tau \bar{\tau}\left[1+a_{2}\left(\mu_{0}^{2}\right) L^{\gamma_{2} / \beta_{0}} C_{2}^{3 / 2}(2 \tau-1)\right]
$$

with the recent lattice QCD result on the second Gegenbauer coefficient [31]

$$
a_{2}\left(\mu_{0}^{2}\right)=0.1364 \pm 0.0213
$$

and the anomalous dimension $\gamma_{2}=50 / 9$. The $1 / \tau$ moment of the twist- 2 pion DA is given by

$$
\langle 1 / \tau\rangle_{\pi}=3\left[1+a_{2}\left(\mu_{R}^{2}\right)\right]
$$

Let us turn to twist-3 contributions. The 2-particle twist-3 contributions were determined in [6], and in this work we rewrite them in a compact form suitable for combining with 3-particle results. We list both 2- and 3-particle twist-3 contribution in Appendix B, while, as we will show, their sum can be simplified and expressed in terms of the convolution with just the 3-particle twist-3 DA. Typical lowest order Feynman diagrams relevant for 3-particle twist-3 contributions are shown in Fig. 2. The 16 Feynman graphs with (c) and without (b) the triplegluon coupling make up the 3-particle contribution. The graphs (c) and (b) have different color factors. Graphs of type (d) for which the constituent gluon of the pion couples to one of its quark constituents are soft contributions and are to be considered as part of the meson wave function. In perturbation theory the gluon field, $A_{\mu}^{a}(x)$, appears in the vacuum-meson matrix elements, whereas the 3-particle DA, $\phi_{3 \pi}$, is defined through the gluon field strength tensor, $G_{\mu \nu}$; see (A7). In the light-cone gauge which we are using, the two quantities are related to each other by [32]

$$
A_{\mu}^{a}(z)=\lim _{\epsilon \rightarrow 0} n^{\nu} \int_{0}^{\infty} d \sigma e^{-\epsilon \sigma} G_{\mu \nu}^{a}(z+n \sigma),
$$

where $n$ is a lightlike vector with $n \cdot A=0$. By making use of this relation and the definition of the 3-particle twist-3 DA (A7) we derive the expression for the vacuum-pion matrix element to be used in the perturbative calculation of the contribution involving $q \bar{q} g$ Fock component

$$
\begin{aligned}
& \left\langle 0\left|\bar{u}^{g}\left(z_{b}\right) A^{\beta, c}\left(z_{g}\right) d^{f}\left(z_{a}\right)\right| \pi^{-}\left(q^{\prime}\right)\right\rangle \\
& =\int[d \tau]_{3} e^{-i q^{\prime} \cdot\left(\tau_{a} z_{a}+\tau_{b} z_{b}+\tau_{g} z_{g}\right)} \mathcal{P}_{3, f g}^{\beta, c}
\end{aligned}
$$

with the 3-particle projector, $q \bar{q} g \rightarrow \pi$, given by

$\mathcal{P}_{3, f g}^{\beta, c}=\frac{i}{g} \frac{f_{3 \pi}}{2 \sqrt{2 N_{C}}} \frac{\left(t^{c}\right)_{f g}}{C_{F} \sqrt{N_{C}}} \frac{\gamma_{5}}{\sqrt{2}} \sigma_{\mu \nu} q^{\prime \mu} g_{\perp}^{\nu \beta} \frac{\phi_{3 \pi}\left(\tau_{a}, \tau_{b}, \tau_{g}\right)}{\tau_{g}}$.

The transverse metric tensor is defined as ${ }^{4}$

$$
g_{\perp}^{\nu \beta}=\left(g^{\nu \beta}-\frac{k_{j}^{\prime \nu} q^{\prime \beta}+q^{\prime \nu} k_{j}^{\prime \beta}}{k_{j}^{\prime} \cdot q^{\prime}}\right)
$$

and the integration measure, $[d \tau]_{3}$, is defined in (A9), while $t^{c}=\lambda^{c} / 2$ is the $\mathrm{SU}(3)$ color matrix for a gluon of color $c$ and $g$ denotes the QCD coupling. As is detailed in Appendix A the equation of motion relates the 2- and 3-particle twist-3 DAs to each other. In the light-cone gauge the relation for the antiquark for instance reads [see (A12) and (A14)]

$$
\begin{gathered}
f_{\pi} \mu_{\pi}\left[\bar{\tau} \phi_{\pi p}(\tau)-\frac{1}{6} \bar{\tau} \phi_{\pi \sigma}{ }^{\prime}(\tau)-\frac{1}{3} \phi_{\pi \sigma}(\tau)\right] \\
=2 f_{3 \pi} \int_{0}^{1-\tau} \frac{d \tau_{g}}{\tau_{g}} \phi_{3 \pi}\left(\tau, \bar{\tau}-\tau_{g}, \tau_{g}\right) .
\end{gathered}
$$

${ }^{4}$ We remind the reader that in our symmetrical c.m.s. the pion and the outgoing quark move back to back, i.e., $\vec{k}_{j}^{\prime}=-\vec{q}^{\prime}$. Transforming $q^{\prime}$ and $k_{j}^{\prime}$ to a frame in which the pion moves along the 3-direction these momenta become

$$
q^{\prime} \rightarrow\left[q^{+}, 0, \overrightarrow{0}_{\perp}\right], \quad k_{j}^{\prime} \rightarrow\left[0, q^{+}, \overrightarrow{0}_{\perp}\right]
$$

with the pion mass being neglected. In this frame the tensor $g_{\perp}^{\nu \beta}$ has the components $g_{\perp}^{11}=g_{\perp}^{22}$ while all other components are zero. 
Using this relation, we can express the full twist-3 subprocess amplitude, the sum of the 2-particle and 3-particle contributions, through the 3-particle DA alone

$$
\begin{aligned}
\mathcal{H}_{0-\lambda, \mu \lambda}^{\mathrm{twist}-3}= & 4 \pi \alpha_{s}\left(\mu_{R}^{2}\right) \frac{f_{3 \pi}\left(\mu_{R}^{2}\right)}{N_{C}}(2 \lambda-\mu) \sqrt{-\frac{\hat{u} \hat{s}}{2}} \frac{1}{\hat{s}^{2} \hat{u}^{2}} \\
& \times \int_{0}^{1} d \tau \int_{0}^{\bar{\tau}} \frac{d \tau_{g}}{\tau_{g}} \phi_{3 \pi}\left(\tau, \bar{\tau}-\tau_{g}, \tau_{g}, \mu_{R}^{2}\right) \\
& \times\left[C_{F}\left(\frac{1}{\bar{\tau}^{2}}-\frac{1}{\bar{\tau}\left(\bar{\tau}-\tau_{g}\right)}\right)\left(\hat{s}^{2}+\hat{u}^{2}\right)\right. \\
& \left.+\left(C_{F}-\frac{1}{2} C_{A}\right)\left(\frac{1}{\tau}+\frac{1}{\bar{\tau}-\tau_{g}}\right) \frac{\hat{t}^{2}}{\tau_{g}}\right],
\end{aligned}
$$

where $C_{A}=N_{C}$. The first term in (33) represents a combination of 2- and 3-particle contributions while the second term is a pure 3-particle contribution. For the interested reader we present the 2- and 3-particle contributions separately in Appendix B. We see from (33) that the twist-3 contribution only feeds the quark helicity-flip subprocess amplitudes in contrast to the twist- 2 contribution which controls the helicity nonflip ones.

We have checked our results by analyzing the gauge invariance conditions. As expected the 2- and 3-particle twist-3 contributions are separately gauge invariant with respect to the choice of gauge of the virtual gluon. In contrast, the gauge invariance with respect to the choice of gauge of the photon is only satisfied by the complete twist-3 result but not separately for the 2- and 3-particle contributions. Another important property of the amplitude (33) is its crossing symmetry. As has been shown long ago [33] the amplitudes $\mathcal{H}_{0-\lambda, \mu \lambda}$ are $\hat{s}-\hat{u}$ crossing symmetric to any order of perturbation theory, ${ }^{5}$ which is evidently the case for (33). As can be seen in Appendix B the separate 2- and 3-particle contributions are not crossing symmetric. Thus, as is evident from the above remarks, both the 2- and the 3-particle twist-3 contributions have to be taken into account in order to obtain a physically consistent result that respects the fundamental properties of gauge invariance and crossing symmetry.

Obviously the 2-particle twist-3 contribution vanishes if the 3-particle DA is assumed to be zero. This has already been noticed in [6]. This situation is to be contrasted with that one in deeply virtual electroproduction of pseudoscalar mesons. In the latter process the contribution from the solution of (A16) for $\phi_{i}^{\mathrm{EOM}}=0$ - the so-called WandzuraWilczek approximation-

$$
\phi_{p}^{W W} \equiv 1, \quad \phi_{\sigma}^{W W}=6 \tau \bar{\tau}
$$

\footnotetext{
${ }^{5}$ The crossing behavior of the amplitude $\mathcal{H}_{0 \lambda, \mu \lambda}$ is more complicated but, as shown in [6], the expression (24) has the correct $\hat{s}-\hat{u}$ crossing property.
}

does not vanish. Up to corrections of order $t / Q^{2}$ where $Q^{2}$ is the virtuality of the photon, the subprocess amplitude in electroproduction is under control of the DA $\phi_{\pi p}$. Because of its end-point behavior it leads to an infrared singularity in collinear approximation. In $[11,12]$ this singularity is regularized by retaining the quark transverse momenta in the subprocess.

The 3-particle DA can be expanded upon the Jacobi polynomials [16]. We employ a truncated version of it:

$$
\begin{aligned}
\phi_{3 \pi}\left(\tau_{a}, \tau_{b}, \tau_{g}, \mu_{R}^{2}\right)= & 360 \tau_{a} \tau_{b} \tau_{g}^{2}\left[1+\omega_{1,0}\left(\mu_{R}^{2}\right) \frac{1}{2}\left(7 \tau_{g}-3\right)\right. \\
& +\omega_{2,0}\left(\mu_{R}^{2}\right)\left(2-4 \tau_{a} \tau_{b}-8 \tau_{g}+8 \tau_{g}^{2}\right) \\
& \left.+\omega_{1,1}\left(\mu_{R}^{2}\right)\left(3 \tau_{a} \tau_{b}-2 \tau_{g}+3 \tau_{g}^{2}\right)\right] .
\end{aligned}
$$

The parameters of the 3-particle DA evolve as

$$
\begin{aligned}
f_{3 \pi}\left(\mu_{R}^{2}\right)= & L^{\left(16 / 3 C_{F}-1\right) / \beta_{0}} f_{3 \pi}\left(\mu_{0}^{2}\right), \\
\omega_{1,0}\left(\mu_{R}\right)= & L^{\left(-25 / 6 C_{F}+11 / 3 C_{A}\right) / \beta_{0}} \omega_{1,0}\left(\mu_{0}^{2}\right), \\
\omega_{11}\left(\mu_{R}^{2}\right)= & \frac{1}{\gamma_{+}-\gamma_{-}}\left[\left(\gamma_{-}-\gamma_{q q}\right) A_{+}\left(\mu_{0}^{2}\right) L^{\left(\gamma_{+}-16 / 3 C_{F}+1\right) / \beta_{0}}\right. \\
& \left.+\left(\gamma_{+}-\gamma_{q q}\right) A_{-}\left(\mu_{0}^{2}\right) L^{\left(\gamma_{-}-16 / 3 C_{F}+1\right) / \beta_{0}}\right], \\
\omega_{20}\left(\mu_{R}^{2}\right)= & \frac{1}{4} \frac{\gamma_{q g}}{\gamma_{-}-\gamma_{+}}\left[A_{+}\left(\mu_{0}^{2}\right) L^{\left(\gamma_{+}-16 / 3 C_{F}+1\right) / \beta_{0}}\right. \\
& \left.+A_{-}\left(\mu_{0}^{2}\right) L^{\left(\gamma_{-}-16 / 3 C_{F}+1\right) / \beta_{0}}\right],
\end{aligned}
$$

where

$$
\begin{aligned}
& A_{+}\left(\mu_{0}^{2}\right)=-\omega_{11}\left(\mu_{0}^{2}\right)-4 \frac{\gamma_{+}-\gamma_{q q}}{\gamma_{q g}} \omega_{20}\left(\mu_{0}^{2}\right), \\
& A_{-}\left(\mu_{0}^{2}\right)=\omega_{11}\left(\mu_{0}^{2}\right)+4 \frac{\gamma_{-}-\gamma_{q q}}{\gamma_{q g}} \omega_{20}\left(\mu_{0}^{2}\right) .
\end{aligned}
$$

The anomalous dimensions are

$\gamma_{q q}=\frac{122}{9}, \quad \gamma_{g g}=\frac{511}{45}, \quad \gamma_{q g}=\frac{5}{3}$,

$\gamma_{g q}=\frac{21}{5}$,

with the eigenvalues

$$
\gamma_{ \pm}=\frac{1}{2}\left[\gamma_{q q}+\gamma_{g g} \pm \sqrt{\left(\gamma_{q q}-\gamma_{g g}\right)^{2}+4 \gamma_{q g} \gamma_{g q}}\right] .
$$

The anomalous dimensions are to be found in the literature $[16,30]$. 
With the help of (35) the integrations in (33) can be performed analytically

$$
\begin{aligned}
\mathcal{H}_{0-\lambda, \mu \lambda}^{\mathrm{twist}-3}= & -80 \pi \alpha_{s} \frac{f_{3 \pi}}{N_{C}}(2 \lambda-\mu) \sqrt{-\frac{\hat{u} \hat{s}}{2}} \frac{1}{\hat{s}^{2} \hat{u}^{2}} \\
& \times\left[C_{F}\left(1-\frac{3}{16} \omega_{1,0}+\frac{6}{25} \omega_{2,0}-\frac{3}{50} \omega_{1,1}\right)\left(\hat{s}^{2}+\hat{u}^{2}\right)\right. \\
& \left.-\left(C_{F}-\frac{1}{2} C_{A}\right)\left(6-\frac{15}{4} \omega_{1,0}+\frac{12}{5} \omega_{2,0}+\frac{3}{5} \omega_{1,1}\right) \hat{t}^{2}\right] .
\end{aligned}
$$

\section{PREDICTIONS FOR PHOTOPRODUCTION OF PSEUDOSCALAR MESONS}

\section{A. The cross section for $\boldsymbol{\pi}^{\mathbf{0}}$ photoproduction}

The most recent determination of the 3-particle pion DA is made in [30] on the basis of QCD sum rules. Instead of $f_{3 \pi}$ the parameter $\eta_{3}$ is quoted in that work. It is related to $f_{3 \pi}$ by (A15). Evolved to the scale $\mu_{0}^{2}$ the value of $\eta_{3}$ derived in [30] leads to

$$
f_{3 \pi}\left(\mu_{0}^{2}\right)=0.004 \mathrm{GeV}^{2} .
$$

The expansion coefficients of the 3-particle DA quoted in [30] are

$$
\omega_{1,0}\left(\mu_{0}^{2}\right)=-2.55, \quad \omega_{2,0}\left(\mu_{0}^{2}\right)=\omega_{1,1}\left(\mu_{0}^{2}\right)=0 .
$$

According to [30] the uncertainties of the parameters (41) and (42) are large, of order of $30 \%$.

We are now in the position to evaluate the photoproduction cross section defined by

$$
\begin{aligned}
\frac{d \sigma}{d t}= & \frac{1}{32 \pi\left(s-m^{2}\right)^{2}}\left[\left|\mathcal{M}_{0+++}\right|^{2}+\left|\mathcal{M}_{0+-+}\right|^{2}\right. \\
& \left.+\left|\mathcal{M}_{0-++}\right|^{2}+\left|\mathcal{M}_{0--+}\right|^{2}\right] .
\end{aligned}
$$

It turns out that with the 3-particle DA specified in Eqs. (41) and (42) the cross section for $\pi^{0}$ photoproduction is still somewhat small as compared to the CLAS data [34]. Since there is no physical reason why $\omega_{20}$ should be zero and its contribution is by no means suppressed as compared to $\omega_{10}$, we fit this parameter to the CLAS data. We obtain

$$
\omega_{20}\left(\mu_{0}^{2}\right)=8.0 \text {. }
$$

This value is a bit smaller than the value quoted in [16,35]. The results of the fit to the $\pi^{0}$ cross section are shown in Fig. 3. The cross section is multiplied by $s^{7}$. This scaling behavior, which holds at a fixed c.m.s. scattering angle $\theta$, follows from dimensional counting for the leading-twist contribution. In order to match roughly the requirement for

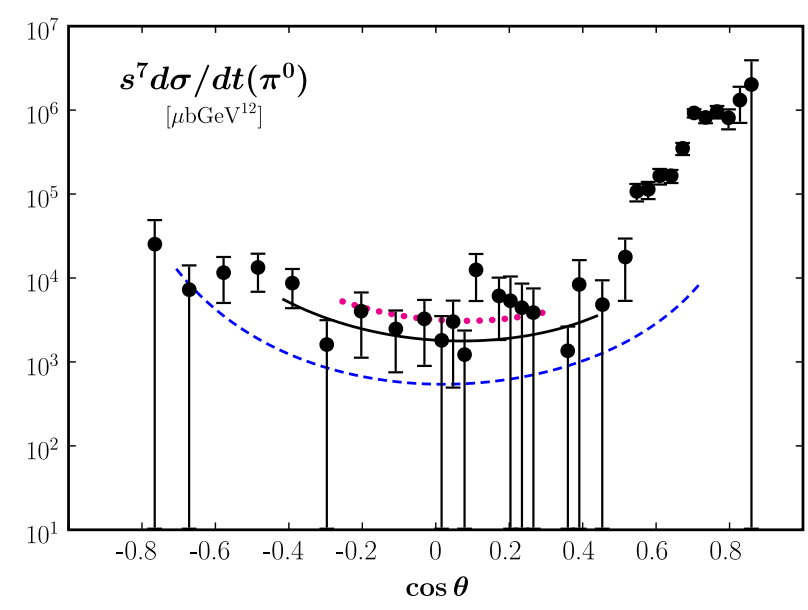

FIG. 3. Results for the cross section of $\pi^{0}$ photoproduction versus the cosine of the c.m.s. scattering angle, $\theta$. The solid (dashed, dotted) curves represent our results at $s=11.06(20,9) \mathrm{GeV}^{2}$. The data at $s=11.06 \mathrm{GeV}^{2}$ are taken from CLAS [34]. The cross sections are multiplied by $s^{7}$, and the theoretical results are only shown for $-t$ and $-u$ larger than $2.5 \mathrm{GeV}^{2}$.

the handbag approach of Mandelstam variables much larger than $\Lambda^{2}$ we only show results for $-t$ and $-u$ larger than $2.5 \mathrm{GeV}^{2}$. As one sees from Fig. 3 our results are in reasonable agreement with the CLAS data [34] at $s=11.06 \mathrm{GeV}^{2}$. For comparison we also present predictions at $s=9$ and $20 \mathrm{GeV}^{2}$. Obviously, the theoretical results drop faster with energy than $s^{-7}$. Leaving aside the $\log$ of $s$ from the evolution the leading-twist handbag results would scale as $s^{-7}$ only if the form factors $R_{V}$ and $R_{A}$ would drop as $1 / t^{2}$ which is not exactly the case; see Table II. In the range of $s$ we are interested in, our cross section effectively behaves $\propto s^{-9}$. This is a consequence of the twist-3 dominance. From the subprocess amplitude one gets a suppression factor $\mu_{\pi}^{2} / s$ in the cross section as compared to the twist-2 contribution; cf. (24) and (33). In addition there are the $\log s$ of $s$ from the evolution of the DAs. The transversity form factors effectively contribute to the energy dependence of the cross section somewhat stronger than $1 / s^{4}$ because their stronger $t$-dependence (see Table II) is only partly compensated by the extra factors of $t$ in the amplitudes (5). Since our form factors represent $1 / x$-moments of GPDs they evolve with the scale in principle. This effect is neglected by us for the following reason: because of the strong $x-t$ correlation the form factors at large $-t$ are under the control of a narrow region of large $x$. With increasing $-t$ this region approaches 1 . Therefore, our form factors become approximately equal to the scale-independent lowest moment of the GPDs concerned (e.g., $R_{V}^{a} \rightarrow F_{1}^{a}$ for $-t \rightarrow \infty$ where $F_{1}^{a}$ is the flavor- $a$ Dirac form factor of the proton). Thus, as it is argued in [18], the $1 / x$-factors in the form factors can be viewed as a phenomenological estimate of effects beyond the strict 
$\Lambda / \sqrt{-t}$ expansion. The evolution effects of the DAs are small. ${ }^{6}$ Since at fixed $s$ the renormalization scale (23) scarcely varies in the wide-angle region, the shape of the theoretical results on the cross section is hardly altered when neglecting the DA evolution, but the effective energy dependence of $d \sigma / d t$ is reduced to about $s^{-8}$. In contrast to electroproduction of the $\pi^{0}$ [12] the cross section is not dominated by single transversity GPDs but the total twist-3 contribution is. For $\pi^{0}$ electroproduction the twist-3 contribution feeds the cross section for transversely polarized photons while twist- 2 controls the longitudinal one. Hence, twist-3 dominance means the dominance of the transverse cross section in $\pi^{0}$ electroproduction which is experimentally confirmed [14] for photon virtualities of the order of $2 \mathrm{GeV}^{2}$. In $\pi^{0}$ photoproduction, on the other hand, both twist- 2 and twist- 3 contribute to the same helicity amplitudes leading to interference terms in the cross section. The twist-2-twist-3 interference term is largest in the forward hemisphere. For $-t \rightarrow 2.5 \mathrm{GeV}^{2}$ it is negative and amounts to $10 \%-15 \%$ in absolute value. In the backward hemisphere the interference term amounts to merely a few percent. Since the twist-3 contribution dominates, the uncertainty of our cross section is correspondingly large. In fact, the parametric uncertainty of the cross section arising from those of the transversity form factors and the twist-3 DA is about $70 \%$ near 90 deg.

As we discuss in Appendix A, the 3-particle twist-3 DA fixes the 2-particle twist-3 DAs through the equations of motion. For the DA (35) with the parameters (41), (42), and (44) the Gegenbauer coefficients of the 2-particle twist-3 DAs are [see Eqs. (A19), (A22), and (A23)]

$$
\begin{array}{ll}
a_{\pi 2}^{p}\left(\mu_{0}^{2}\right)=-0.56, & a_{\pi 4}^{p}\left(\mu_{0}^{2}\right)=0.17, \\
a_{\pi 2}^{\sigma}\left(\mu_{0}^{2}\right)=-0.084, & a_{\pi 4}^{\sigma}\left(\mu_{0}^{2}\right)=0.031
\end{array}
$$

$\left(a_{\pi n}^{p}=a_{\pi n}^{\sigma}=0\right.$ for $\left.n \geq 4\right)$, and

$$
\eta_{\sigma}\left(\mu_{0}^{2}\right)=0.64 \text {. }
$$

The values of the Gegenbauer coefficients $a_{\pi 2}^{p}$ and, with regard to the value of $\eta_{\sigma}$, also that of $a_{\pi 2}^{\sigma}$ are compatible with those to be found in the literature while the coefficients $a_{\pi 4}^{p(\sigma)}$ have opposite signs. These 2-particle twist-3 DAs from the literature have been derived with various methods: the Dyson-Schwinger approach [36], a light-cone quark model [37], and a chiral quark model [38]. The 3-particle DA is not considered in these papers, and therefore no result on $f_{3 \pi}$ is quoted. However, this parameter plays an

\footnotetext{
${ }^{6}$ Taking the parameters $f_{3 \pi}=0.005 \mathrm{GeV}^{2}, \omega_{1,0}=-3$, $\omega_{2,0}=7$, and $\omega_{1,1}=0$, valid at a low scale of about $1 \mathrm{GeV}$ and ignoring evolution, the predictions for the photoproduction cross section at $s=11.06 \mathrm{GeV}^{2}$ are almost indistinguishable from that one shown in Fig. 3.
}

important role in the present work. Our values for the Gegenbauer coefficients of $\phi_{\pi p}$ (45) have opposite signs to those quoted in [30]. The latter Gegenbauer coefficients have been derived from the same 3-particle DA that we are using, but the Fock-Schwinger gauge is employed in the vacuum-meson matrix elements. Thus, the different methods applied in [30] and by us lead to drastic differences in the 2-particle twist-3 DAs. The normalization $\eta_{\sigma}$ may be absorbed into, say, the mass parameter $\mu_{\pi}$ in the case of the DA $\phi_{\pi \sigma}$ leading to a mass parameter $\mu_{\pi \sigma}$ which is somewhat smaller than the mass parameter $\mu_{\pi}$ appearing for $\phi_{\pi p}$. In [29] it is claimed that such differences in the mass parameter may be generated by the off-shellness of the quarks and antiquarks in the pion.

\section{B. Spin effects}

The derivation of the photoproduction amplitudes within the handbag approach naturally requires the use of the light-cone helicity basis. However, for comparison with experimental results on spin-dependent observables, the use of the ordinary photon-proton c.m.s. helicity basis is more convenient. The standard helicity amplitudes, $\Phi_{0 \nu^{\prime}, \mu \nu}$, are obtained from the light-cone helicity amplitudes (5), by the transform [8]

$$
\begin{aligned}
\Phi_{0 \nu^{\prime}, \mu \nu}= & \mathcal{M}_{0 \nu^{\prime}, \mu \nu}+\frac{1}{2} \kappa\left[(-1)^{1 / 2-\nu^{\prime}} \mathcal{M}_{0-\nu^{\prime}, \mu \nu}\right. \\
& \left.+(-1)^{1 / 2+\nu} \mathcal{M}_{0 \nu^{\prime}, \mu-\nu}\right]+\mathcal{O}\left(m^{2} / s\right),
\end{aligned}
$$

where

$$
\kappa=\frac{2 m}{\sqrt{s}} \frac{\sqrt{-t}}{\sqrt{s}+\sqrt{-u}} .
$$

For convenience the notation for the helicities is kept. Obviously,

$$
\sum_{\nu^{\prime}, \mu}\left|\Phi_{0 \nu^{\prime}, \mu+}\right|^{2}=\sum_{\nu^{\prime}, \mu}\left|\mathcal{M}_{0 \nu^{\prime}, \mu+}\right|^{2}
$$

As for wide-angle Compton scattering [20,39] the most interesting spin-dependent observables are the correlations of the helicities of the incoming photon and the incoming, $A_{\mathrm{LL}}$, or outgoing proton, $K_{\mathrm{LL}}$ :

$$
\begin{aligned}
& A_{\mathrm{LL}}=\frac{\left|\Phi_{0+,++}\right|^{2}-\left|\Phi_{0+,-+}\right|^{2}+\left|\Phi_{0-,++}\right|^{2}-\left|\Phi_{0-,-+}\right|^{2}}{\sum_{\nu^{\prime}, \mu}\left|\Phi_{0 \nu^{\prime}, \mu+}\right|^{2}}, \\
& K_{\mathrm{LL}}=\frac{\left|\Phi_{0+,++}\right|^{2}-\left|\Phi_{0+,-+}\right|^{2}-\left|\Phi_{0-,++}\right|^{2}+\left|\Phi_{0-,-+}\right|^{2}}{\sum_{\nu^{\prime}, \mu}\left|\Phi_{0 \nu^{\prime}, \mu+}\right|^{2}} .
\end{aligned}
$$

One can easily check that for the twist-3 contribution one has 


$$
A_{\mathrm{LL}}^{\mathrm{twist}-3}=-K_{\mathrm{LL}}^{\mathrm{twist}-3}
$$

while for the twist-2 contribution

$$
A_{\mathrm{LL}}^{\mathrm{twist}-2}=K_{\mathrm{LL}}^{\mathrm{twist}-2}
$$

holds as is the case for wide-angle Compton scattering [20,39]. Thus, the helicity correlations may provide a characteristic signal for the dominance of twist-3 contribution in photoproduction of pseudoscalar mesons. Thus the observables $A_{\mathrm{LL}}$ and $K_{\mathrm{LL}}$ play a similar important role for the discrimination between twist-2 and twist-3 in photoproduction of pions as the longitudinal and transverse cross sections in pion electroproduction.

In terms of helicity amplitudes the correlation between the helicity of the photon and the sideways polarization of the incoming proton is ${ }^{7}$

$$
A_{\mathrm{LS}}=2 \frac{\operatorname{Re}\left[\Phi_{0+,++}^{*} \Phi_{0-,-+}-\Phi_{0+,-+}^{*} \Phi_{0-,++}\right]}{\sum_{\nu^{\prime}, \mu}\left|\Phi_{0 \nu^{\prime}, \mu+}\right|^{2}},
$$

and the correlation between the helicity of the photon and the sideways polarization of the recoil proton is

$$
K_{\mathrm{LS}}=2 \frac{\operatorname{Re}\left[\Phi_{0+,++}^{*} \Phi_{0-,++}-\Phi_{0+,-+}^{*} \Phi_{0-,-+}\right]}{\sum_{\nu^{\prime}, \mu}\left|\Phi_{0 \nu^{\prime}, \mu+}\right|^{2}} .
$$

The last spin observable we consider is the asymmetry for linearly polarized photons, transverse and parallel to the photon momentum,

$$
\Sigma=2 \frac{\operatorname{Re}\left[\Phi_{0+,++}^{*} \Phi_{0+,-+}+\Phi_{0-,++}^{*} \Phi_{0-,-+}\right]}{\sum_{\nu^{\prime}, \mu}\left|\Phi_{0 \nu^{\prime}, \mu+}\right|^{2}}
$$

Since the twist- 3 subprocess amplitude, $\mathcal{H}_{0-,++}^{\text {twist }-3}$, is zero as can be seen from (33), any spin observable is only given by a ratio of the transversity form factors up to corrections from twist-2. Hence, the predictions on spin-dependent observables are more precise than those for the cross sections since only the uncertainties of the form factors matter. Consequently, they do not suffer from the large uncertainties arising from the 3-particle DA as is the case for the differential cross section.

It is instructive to quote the observables obtained from the twist- 3 contribution alone since this is the dominant contribution. In this case the cross section is given by

$$
\frac{d \sigma^{\text {twist-3 }}}{d t}=\frac{\pi \alpha_{\mathrm{em}}}{32\left(s-m^{2}\right)^{2}}\left|\mathcal{H}_{0-,-+}^{\mathrm{twist}-3}\right|^{2} F^{\pi^{0}}
$$

where the combination of form factors, $F^{\pi^{0}}$, reads

\footnotetext{
${ }^{7}$ Sideways is defined as the direction perpendicular to the proton momentum but in the scattering plane.
}

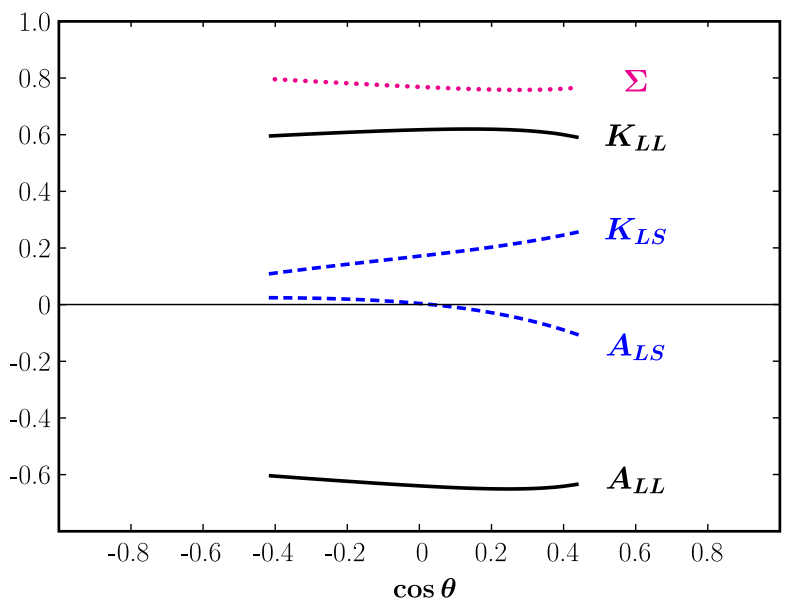

FIG. 4. Predictions for spin observables of $\pi^{0}$ photoproduction at $s=11.06 \mathrm{GeV}^{2}$. The parametric uncertainty is $\simeq 15 \%$ near $90 \mathrm{deg}$.

$$
F^{\pi^{0}}=-\frac{t}{2 m^{2}}\left[\bar{S}_{T}^{\pi^{0} 2}-\frac{t}{m^{2}} S_{S}^{\pi^{0} 2}+4 S_{S}^{\pi^{0}} S_{T}^{\pi^{0}}-8 \frac{m^{2}}{t} S_{T}^{\pi^{0} 2}\right]
$$

The spin-dependent observables then read

$$
\begin{aligned}
& A_{\mathrm{LL}}^{\mathrm{twist}-3}=-K_{\mathrm{LL}}^{\mathrm{twist}-3}=-4 \frac{S_{T}^{\pi^{0}}\left[S_{T}^{\pi^{0}}-\frac{t}{2 m^{2}} S_{S}^{\pi^{0}}+\kappa \frac{\sqrt{-t}}{2 m} \bar{S}_{T}^{\pi^{0}}\right]}{F^{\pi^{0}}}, \\
& \left.A_{L S}^{\mathrm{twist}-3}=-K_{L S}^{\mathrm{twist}-}-3\right) \frac{S_{T}^{\pi^{0}}}{F^{\pi^{0}}}\left[\frac{\sqrt{-t}}{m} \bar{S}_{T}^{\pi^{0}}-2 \kappa\left(S_{T}^{\pi^{0}}-\frac{t}{2 m^{2}} S_{S}^{\pi^{0}}\right)\right] \\
& \Sigma^{\mathrm{twist}-3}=1-4 \frac{S_{T}^{\pi^{0}}}{F^{\pi^{0}}} .
\end{aligned}
$$

Since only the form factors are needed in (58), it seems possible to give predictions of such observables for different meson channels. It is also evident from (58) that these spin observables are independent on energy at fixed $t$ up to corrections from twist-2 and corrections of order $\Lambda^{2} / s$.

In Fig. 4 we show predictions on the spin-dependent observables for $\pi^{0}$ photoproduction. One sees that $A_{\mathrm{LL}}$ and $K_{\mathrm{LL}}$ are large in absolute value and almost mirror symmetrical. The observables $A_{\mathrm{LS}}$ and $K_{\mathrm{LS}}$ are small in absolute value. The twist- 2 contributions to them are relatively large. The observable $\Sigma$ is close to unity and only mildly $t$-dependent. In tendency this is in agreement with a glueX measurement for $\pi^{0}$ photoproduction at small $-t$ [40].

At Jefferson Lab the observables $K_{\mathrm{LL}}$ and $K_{L S}$ have been measured twice: at $s=7.8 \mathrm{GeV}^{2}$ and a c.m.s. scattering angle of $70 \mathrm{deg}$ [41] and at $s=6.9 \mathrm{GeV}^{2}$ and $\theta=120^{\circ}$ [42]. These kinematical settings do not respect the requirement of large Mandelstam variables; either $-t$ or $-u$ is too small. The data are as follows: 


$$
\begin{aligned}
{[41] \quad s } & =7.8 \mathrm{GeV}^{2}, \quad t=-2.1 \mathrm{GeV}^{2}: \\
K_{\mathrm{LL}} & =-0.082 \pm 0.007, \quad K_{L S}=-0.296 \pm 0.007 \\
{[42] \quad s } & =6.9 \mathrm{GeV}^{2}, \quad u=-1.04 \mathrm{GeV}^{2}: \\
K_{\mathrm{LL}} & =0.532 \pm 0.006, \quad K_{L S}=0.480 \pm 0.007
\end{aligned}
$$

Inspection of Fig. 4 shows that the data at $6.9 \mathrm{GeV}^{2}$ agree with our predictions in tendency while the $7.8 \mathrm{GeV}^{2}$ data are smaller. This is similar to the situation in wide-angle Compton scattering [20]. Before conclusions can be drawn we have to wait for data at $s,-t,-u \gg \Lambda^{2}$. Such data are planned to be measured at Jefferson Lab [43].

\section{Other channels}

In this section we are going to comment briefly on other wide-angle photoproduction processes. From the theoretical point of view the simplest case is of course $\pi^{0}$ photoproduction off neutrons. In this case we only have to change the process form factors. By isospin invariance the form factors are now

$$
F_{\text {in }}^{\pi^{0}}(t)=\frac{1}{\sqrt{2}}\left[e_{u} F_{i}^{d}(t)-e_{d} F_{i}^{u}(t)\right]
$$

instead of (12). Predictions for the corresponding cross section are shown in Fig. 5. They are about a factor of 2.5 smaller than those for $\pi^{0}$ photoproduction off protons. For very large $-t$ the ratio of cross sections for $\pi^{0}$ photoproduction off neutrons and off protons becomes equal to $\left(e_{d} / e_{u}\right)^{2}$ since, according to Table II, the $d$-quark form factors drop faster with increasing $-t$ than the $u$-quark ones. Consequently, the $d$-quark form factors can be neglected at large $-t$. Spin effects are similar to those

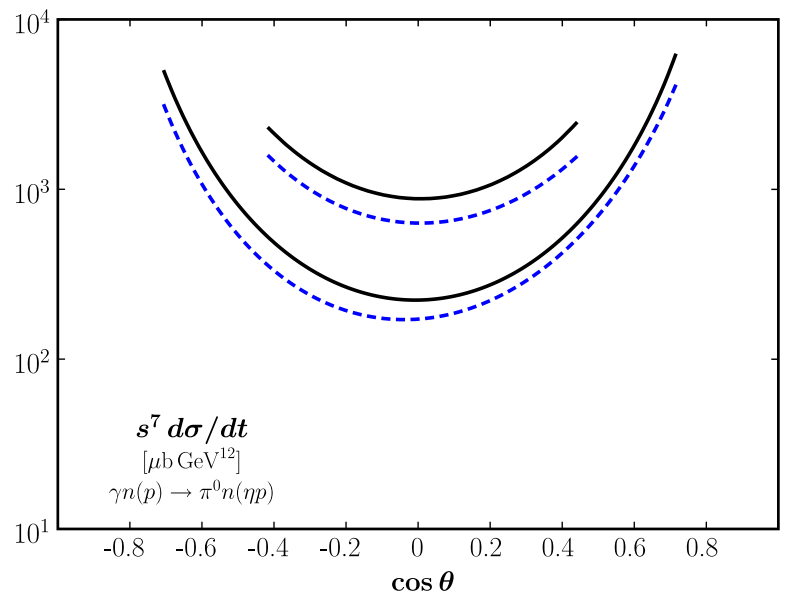

FIG. 5. Predictions for the cross sections of $\pi^{0}$ photoproduction off neutrons (solid lines) and $\eta$ photoproduction (dashed lines) at $s=11.06$ (upper lines) and $20 \mathrm{GeV}^{2}$ (lower lines). The parametric uncertainty amounts to about $70 \%$ near $90 \mathrm{deg}$. for the case of a proton target; see Fig. 4. The observables $A_{\mathrm{LL}}, K_{\mathrm{LL}}, A_{\mathrm{LS}}$, and $K_{\mathrm{LS}}$ are merely somewhat smaller in absolute value than the corresponding observables measured with a proton target.

For $\eta$ photoproduction off protons the situation is more complex as is detailed in [12]. The mixing of the $\eta$ and $\eta^{\prime}$ is to be taken into account, and the form factors for strange quarks are also needed in principle. However, for the charge-conjugation even mesons the GPDs only contribute in the valence quark (or flavor nonsinglet) combination $F_{i}^{a}-F_{i}^{\bar{a}}$. For the strange quark it seems to be plausible to assume $F_{i}^{s} \simeq F_{i}^{\bar{s}}$ [3]. Hence, there is no contribution from strange quarks and, as is discussed in [12], the flavor-octet and singlet form factors are approximately given by

$$
F_{i}^{(8)}(t) \simeq \frac{1}{\sqrt{2}} F_{i}^{(1)}(t) \simeq \frac{1}{\sqrt{6}}\left[e_{u} F_{i}^{u}(t)+e_{d} F_{i}^{d}(t)\right]
$$

Using the mixing scheme advocated for in [44], one can also decompose the $\eta$ amplitudes in a flavor-octet and single part

$$
\mathcal{M}_{i}^{\eta}=\cos \theta_{8} \mathcal{M}_{i}^{(8)}-\sin \theta_{1} \mathcal{M}_{i}^{(1)}
$$

with the mixing angles

$$
\theta_{8}=-21.2^{\circ}, \quad \theta_{1}=-9.2^{\circ},
$$

derived in [44] on exploiting the divergences of the axialvector current. Assuming furthermore that the octet and singlet DAs, for both twist-2 and twist-3, are the same as the pion DAs and taking the values ${ }^{8}$

$$
f_{8} \simeq 1.26 f_{\pi},[44] \quad f_{3 \eta}^{(8)}=0.87 f_{3 \pi},[30]
$$

for the decay constants, one finds for the $\eta$ amplitude

$$
\mathcal{M}_{i}^{\eta} \simeq \mathcal{M}_{i}^{(8)}\left(\cos \theta_{8}-\sqrt{2} \sin \theta_{1}\right) .
$$

In principle there is also a contribution from the two-gluon Fock component of the $\eta$ [27]. Since this contribution possesses flavor-singlet quantum numbers and is of leading-twist nature, it can safely be neglected. Predictions for $\eta$ photoproduction cross section are shown in Fig. 5. The $\eta$ cross section is similar in shape to the $\pi^{0}$ one but about a factor of 2 smaller than the cross section for $\pi^{0}$ production off neutrons.

In Fig. 6 we present predictions on the helicity correlations, $A_{\mathrm{LL}}$ and $K_{\mathrm{LL}}$, for $\pi^{0}$ photoproduction off neutrons and for $\eta$ photoproduction off proton.

It is also possible to calculate observables for the photoproduction of charged pions and kaons. In these

\footnotetext{
${ }^{8}$ For the mass parameter $\mu_{\eta}^{(8)}$ one may take approximately the same value as for $\mu_{\pi}$ [12].
} 


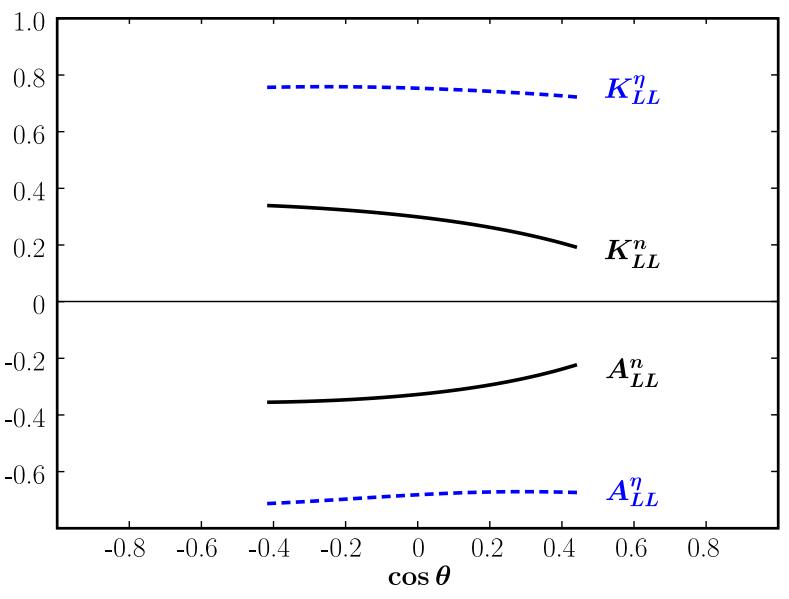

FIG. 6. Predictions for spin observables of $\pi^{0}$ photoproduction off neutrons and of $\eta$ production at $s=11.06 \mathrm{GeV}^{2}$. The parametric uncertainty is $\simeq 15 \%$ near $90 \mathrm{deg}$.

reactions transition GPDs and form factors appear which, due to flavor symmetry, are related to the proton-proton ones [45]. For instance the form factors for photoproduction of charged pions are given by

$$
F_{i p \rightarrow n}^{\pi}=F_{i n \rightarrow p}^{\pi}=F_{i}^{u}-F_{i}^{d} .
$$

Since this combination of flavor form factors is not well known, we refrain from presenting predictions for the corresponding cross sections. A new feature for the kaon-hyperon channels is the appearance of form factors for strange quarks which, at large $-t$, are unknown as yet. Moreover, the 3-particle DA for the kaon is also needed which is not well known [46]. We therefore refrain from giving predictions for these channels too. We stress, however, that from data on these channels one may extract information on the form factors and the twist-3 DAs. For instance, from spin-dependent observables one may learn about the form factors for strange quarks and subsequently from the differential cross section on the 3-particle DA of the kaon.

\section{SUMMARY}

We have calculated wide-angle photoproduction of $\pi^{0}$ mesons within the handbag factorization scheme to twist-3 accuracy. The twist- 3 contribution includes both the 2-particle, $q \bar{q}$, as well as the 3-particle, $q \bar{q} g$, parts. In the light-cone gauge which we are using for the vacuummeson matrix elements, the equation of motion enables us to express the 2-particle twist-3 DAs through an integral upon the 3-particle DA. This relation is formally an inhomogeneous linear differential equation of first order which can readily be solved for a given 3-particle DA. The use of the light-cone gauge made it also possible to derive a compact 3-particle twist-3 projector in momentum space. Our twist-3 subprocess amplitude respects gauge invariance in QCD and QED and $\hat{s}-\hat{u}$ crossing symmetry. On the other hand, the separate 2- and 3-particle twist-3 amplitudes do not possess these properties. This reveals the necessity of taking into account both the 2-and 3 -particle contributions in order to obtain a physically consistent result. Our calculation method for the subprocess amplitudes is similar to a one exploited in [24,25] in a calculation of electroproduction of transversely polarized $\rho$-mesons. We emphasize that the twist- 3 effect we considered which follows from the twist-3 pion DA in conjunction with leading-twist transversity GPDs, is very strong due to the large mass parameter $\mu_{\pi}$. Twist- 3 effects may also be generated by twist-3 GPDs [47]. However, for these GPDs there is no similar enhancement known. Therefore, the contribution from the twist-3 GPDs is expected to be small and neglected by us.

With the help of the relation between the 2-particle DAs, $\phi_{p}$ and $\phi_{\sigma}$, and the 3-particle DA, $\phi_{3 \pi}$, the twist-3 subprocess amplitude can solely be expressed by the latter DA. This manifestly demonstrates the vanishing of the twist-3 contribution in the Wandzura-Wilczek approximation which we observed previously [6]. For the numerical analysis we thus have only to specify the 3-particle DA. The parameters $f_{3 \pi}$ and $\omega_{10}$ are taken from the literature [30]. A third parameter, $\omega_{20}$, is fitted to the recent CLAS data on $\pi^{0}$ photoproduction at $s=11.06 \mathrm{GeV}^{2}$ [34]. The twist-2 subprocess amplitude is taken from our previous work [6], and for the twist-2 DA we used recent lattice gauge theory results [31]. The form factors, $R_{V}$ and $R_{T}$, representing $1 / x$-moments of GPDs, are taken from the GPD analysis of the electromagnetic form factors of the nucleon [3] while for the form factor $R_{A}$ a result advocated for in [20] is employed. The transversity form factors, $S_{T}$, $\bar{S}_{T}$, and $S_{S}$, are evaluated from the transversity GPDs discussed in [12] which describe fairly well exclusive electroproduction of pions at small $-t$. These GPDs are extrapolated to the large $-t$ region.

Our results for the $\pi^{0}$ cross section agree rather well with the recent CLAS data [34]. It turns out that the twist-3 contribution dominates by far, and the twist 2 contribution is almost negligible. Thus, we observed the same situation for wide-angle $\pi^{0}$ photoproduction as for deeply virtual $\pi^{0}$ electroproduction. We also presented predictions for the cross section at other energies and for a number of spindependent observables. Particularly noteworthy are the helicity correlations $A_{\mathrm{LL}}$ and $K_{\mathrm{LL}}$. In contrast to wideangle Compton scattering where $A_{\mathrm{LL}}=K_{\mathrm{LL}}$ [39] they are nearly mirror symmetric (i.e., $A_{\mathrm{LL}} \simeq-K_{\mathrm{LL}}$ ) in wide-angle photoproduction of $\pi^{0}$ or $\eta$ mesons. This result is a consequence of the twist-3 dominance.

The twist-3 mechanism we have proposed applies to the $s-t$ crossed process, $p \bar{p} \rightarrow \gamma \pi^{0}$, too. Also for that process the twist-2 contribution falls short in comparison with experiment [48]. A hint at a dominant higher-twist contribution to this process comes from the FERMI lab E760 
experiment [49] which clearly has a stronger energy dependence than predicted by dimensional counting.

\section{ACKNOWLEDGMENTS}

We are grateful to Han Wen Huang who collaborated with us at an early stage of the work. We also would like to thank Volodya Braun, Dimitry Ivanov, Rainer Jakob, Dieter Müller, Lech Szymanowski, and Samuel Wallon for discussions and comments. This work has been supported in part by the Croatian Science Foundation (HrZZ) project "Physics of Standard Model and beyond" HrZZ 5169, and the H2020 CSA Twinning Project No. 692194, "RBI-TWINNING."

\section{APPENDIX A: THE 2- AND 3-PARTICLE TWIST-3 PION DAs}

In this section we supplement the main part of the paper by summarizing the definitions of the 2- and 3-particle pion distribution amplitudes up to twist-3 $[16,30,50]$ and the equations that relate them. For notational convenience we quote the vacuum-pion matrix elements for a charged pion. Their generalization to the case of a $\pi^{0}$ is obvious. One has to write the quark field operators as

$$
\frac{1}{\sqrt{2}}\left\langle 0|\bar{u} \Gamma u-\bar{d} \Gamma d| \pi^{0}\right\rangle .
$$

All that we present in this appendix can straightforwardly be generalized to other pseudoscalar mesons.

The twist-2 DA is defined by the vacuum-pion matrix element

$$
\begin{aligned}
& \left\langle 0\left|\bar{u}\left(z_{2}\right) \gamma_{\mu} \gamma_{5} d\left(z_{1}\right)\right| \pi^{-}\left(q^{\prime}\right)\right\rangle \\
& \quad=i f_{\pi} q_{\mu}^{\prime} \int_{0}^{1} d \tau e^{-i\left(\bar{\tau} q^{\prime} \cdot z_{2}+\tau q^{\prime} \cdot z_{1}\right)} \phi_{\pi}(\tau) .
\end{aligned}
$$

The 2-particle twist-3 DAs are defined by

$$
\begin{aligned}
& \left\langle 0\left|\bar{u}\left(z_{2}\right) \gamma_{5} d\left(z_{1}\right)\right| \pi^{-}\left(q^{\prime}\right)\right\rangle \\
& =i f_{\pi} \mu_{\pi} \int_{0}^{1} d \tau e^{-i\left(\bar{\tau} q^{\prime} \cdot z_{2}+\tau q^{\prime} \cdot z_{1}\right)} \phi_{\pi p}(\tau), \\
& \left\langle 0\left|\bar{u}\left(z_{2}\right) \sigma_{\mu \nu} \gamma_{5} d\left(z_{1}\right)\right| \pi^{-}\left(q^{\prime}\right)\right\rangle \\
& =\frac{i}{6} f_{\pi} \mu_{\pi}\left(q_{\mu}^{\prime} z_{\nu}-q_{\nu}^{\prime} z_{\mu}\right) \\
& \quad \times \int_{0}^{1} d \tau e^{-i\left(\bar{\tau} q^{\prime} \cdot z_{2}+\tau q^{\prime} \cdot z_{1}\right)} \phi_{\pi \sigma}(\tau) .
\end{aligned}
$$

Here $z=z_{2}-z_{1}\left(z=\left[0, z^{-}, 0_{\perp}\right]\right)$ and we take the massless limit, $q^{\prime 2}=0$. We remind the reader that we are working in light-cone gauge. All Wilson lines, i.e., the path-ordered exponentials of the gluon fields, are unity in that gauge. The local limits of the $\gamma_{\mu} \gamma_{5}$ and $\gamma_{5}$ matrix elements

$$
\begin{aligned}
\left\langle 0\left|\bar{u}(0) \gamma_{\mu} \gamma_{5} d(0)\right| \pi^{-}\left(q^{\prime}\right)\right\rangle & =i f_{\pi} q_{\mu}^{\prime}, \\
\left\langle 0\left|\bar{u}(0) \gamma_{5} d(0)\right| \pi^{-}\left(q^{\prime}\right)\right\rangle & =i f_{\pi} \mu_{\pi}
\end{aligned}
$$

provide constraints on the DAs $\phi_{\pi}$ and $\phi_{\pi p}$,

$$
\int_{0}^{1} d \tau \phi_{\pi}(\tau)=\int_{0}^{1} d \tau \phi_{\pi p}(\tau)=1
$$

as one sees with the help of translation invariance. There is no such constraint on $\phi_{\pi \sigma}$. Its normalization is fixed by the equation of motion as we will see below. The definitions (A2) and (A3) can be combined into

$$
\begin{aligned}
& \left\langle 0\left|\bar{u}^{g}\left(z_{2}\right) d^{f}\left(z_{1}\right)\right| \pi^{-}\left(q^{\prime}\right)\right\rangle \\
& =\frac{i f_{\pi}}{4} \frac{\delta_{f g}}{N_{C}} \int_{0}^{1} d \tau e^{-i\left(\bar{\tau} q^{\prime} \cdot z_{2}+\tau q^{\prime} \cdot z_{1}\right)} \\
& \quad \times\left\{\gamma_{5} q^{\prime} \phi_{\pi}+\mu_{\pi} \gamma_{5}\left[\phi_{\pi p}-\sigma_{\mu \nu} q^{\prime \mu} x^{\nu} \phi_{\pi \sigma}\right]\right\},
\end{aligned}
$$

leading to (19).

The 3-particle twist-3 DA is defined by the quarkantiquark-gluon vacuum-pion matrix element

$$
\begin{aligned}
& \left\langle 0\left|\bar{u}\left(z_{b}\right) \sigma_{\mu \nu} \gamma_{5} g G_{\alpha \beta}\left(z_{g}\right) d\left(z_{a}\right)\right| \pi\left(q^{\prime}\right)\right\rangle \\
& =i f_{3 \pi}\left[q_{\alpha}^{\prime}\left(q_{\mu}^{\prime} g_{\nu \beta}-q_{\nu}^{\prime} g_{\mu \beta}\right)-(\alpha \leftrightarrow \beta)\right] \\
& \quad \times \int[d \tau]_{3} e^{-i\left(z_{a} \tau_{a}+z_{b} \tau_{b}+z_{g} \tau_{g}\right)} \phi_{3 \pi}\left(\tau_{a}, \tau_{b}, \tau_{g}\right),
\end{aligned}
$$

where

$$
[d \tau]_{3}=d \tau_{a} d \tau_{b} d \tau_{g} \delta\left(1-\tau_{a}-\tau_{b}-\tau_{g}\right) .
$$

As usual also the 3-particle DA is normalized to

$$
\int[d \tau]_{3} \phi_{3 \pi}\left(\tau_{a}, \tau_{b}, \tau_{g}\right)=1 .
$$

The 2-particle and 3-particle twist-3 DAs are connected through the equations of motion:

$$
\begin{aligned}
0= & \left\langle 0\left|\bar{u}\left(z_{2}\right) i \varnothing_{z_{1}} d\left(z_{1}\right)\right| \pi^{-}\left(q^{\prime}\right)\right\rangle \\
= & \left\langle 0\left|\bar{u}\left(z_{2}\right)\left(i \varnothing_{z_{1}}\right) d\left(z_{1}\right)\right| \pi^{-}\left(q^{\prime}\right)\right\rangle \\
& +\langle 0| \bar{u}\left(z_{2}\right) X\left(\left(z_{1}\right) d\left(z_{1}\right)\left|\pi^{-}\left(q^{\prime}\right)\right\rangle .\right.
\end{aligned}
$$

An analogous equation holds for the antiquark field. Using (A6) and (29), derived for the light-cone gauge, we cast the equations of motion into the simple closed form

$$
\tau \phi_{\pi p}(\tau)+\frac{1}{6} \tau \phi_{\pi \sigma}^{\prime}(\tau)-\frac{1}{3} \phi_{\pi \sigma}(\tau)=\phi_{1}^{\mathrm{EOM}}(\tau),
$$

and for the antiquark

$$
\bar{\tau} \phi_{\pi p}(\tau)-\frac{1}{6} \bar{\tau} \phi_{\pi \sigma}^{\prime}(\tau)-\frac{1}{3} \phi_{\pi \sigma}(\tau)=\phi_{2}^{\mathrm{EOM}}(\tau),
$$

where 


$$
\phi_{1}^{\mathrm{EOM}}(\tau)=2 \eta_{3} \int_{0}^{\tau} \frac{d \tau_{g}}{\tau_{g}} \phi_{3 \pi}\left(\tau-\tau_{g}, \bar{\tau}, \tau_{g}\right)
$$

and

$$
\phi_{2}^{\mathrm{EOM}}(\tau)=2 \eta_{3} \int_{0}^{\bar{\tau}} \frac{d \tau_{g}}{\tau_{g}} \phi_{3 \pi}\left(\tau, \bar{\tau}-\tau_{g}, \tau_{g}\right) .
$$

The prefactor $\eta_{3}$ is defined by

$$
\eta_{3}=\frac{f_{3 \pi}}{f_{\pi} \mu_{\pi}}
$$

We stress that both the 2- and the 3-particle pion DAs are symmetric under the exchange of the quark and antiquark momentum fractions; (A13) and (A14) are related by the replacement $\tau \leftrightarrow \bar{\tau}$. Similar relations as (A11) and (A12) have been derived in the light-cone gauge for the twist- 3 DAs of a transversely polarized $\rho$ meson in [24].

A suitable combination of (A11) and (A12) leads to a first order linear differential equation for the DA $\phi_{\sigma}$ which can easily be solved. The other DA, $\phi_{p}$, can subsequently be determined from (A11) for instance. We find for the 2-particle twist-3 DAs

$\phi_{\pi \sigma}(\tau)=6 \tau \bar{\tau}\left(\int d \tau \frac{\bar{\tau} \phi_{1}^{\mathrm{EOM}}(\tau)-\tau \phi_{2}^{\mathrm{EOM}}(\tau)}{2 \tau^{2} \bar{\tau}^{2}}+C\right)$,

$\phi_{\pi p}(\tau)=\frac{1}{6 \tau \bar{\tau}} \phi_{\pi \sigma}(\tau)+\frac{1}{2 \tau} \phi_{1}^{\mathrm{EOM}}(\tau)+\frac{1}{2 \bar{\tau}} \phi_{2}^{\mathrm{EOM}}(\tau)$.

Using the 3-particle DA (35) and fixing the constant of integration, $C$, such that the constraint (A5) on $\phi_{\pi p}$ is respected, we find

$$
C=\left[1+\eta_{3}\left(7 \omega_{1,0}-2 \omega_{2,0}-\omega_{1,1}\right)\right]
$$

and

$$
\phi_{\pi p}=1+\sum_{n=2,4, \ldots} a_{\pi n}^{p} C_{n}^{(1 / 2)}(2 \tau-1)
$$

with the Gegenbauer coefficients $\left(a_{\pi n}^{p}=0\right.$ for $n \geq 6$ )

$$
a_{\pi 2}^{p}=-\frac{10}{3} a_{\pi 4}^{p}=\frac{10}{7} \eta_{3}\left(7 \omega_{1,0}-2 \omega_{2,0}-\omega_{1,1}\right) .
$$

Obviously, the second 2-particle twist-3 DA is not normalized to unity but we achieve that by a renormalization

$$
\phi_{\pi \sigma}=\eta_{\sigma} \tilde{\phi}_{\pi \sigma}
$$

with

$$
\tilde{\phi}_{\pi \sigma}=6 \tau \bar{\tau}\left[1+\sum_{n=2,4, \ldots} a_{\pi n}^{\sigma} C_{n}^{(3 / 2)}(2 \tau-1)\right] .
$$

In this case the Gegenbauer coefficients read $\left(a_{\pi n}^{\sigma}=0\right.$ for $n \geq 6$ )

$$
\begin{aligned}
& a_{\pi 2}^{\sigma}=\frac{1}{6} \frac{\eta_{3}}{\eta_{\sigma}}\left(12+3 \omega_{1,0}-4 \omega_{2,0}\right), \\
& a_{\pi 4}^{\sigma}=\frac{1}{105} \frac{\eta_{3}}{\eta_{\sigma}}\left(22 \omega_{2,0}-3 \omega_{1,1}\right),
\end{aligned}
$$

and with

$$
\eta_{\sigma}=1-\eta_{3}\left(12-4 \omega_{1,0}+\frac{8}{7} \omega_{2,0}+\frac{4}{7} \omega_{1,1}\right) .
$$

In the limit $\eta_{3} \rightarrow 0$, i.e., if the 3-particle DA is ignored, the 2-particle twist-3 DAs reduce to the Wandzura-Wilczek approximation (34)

$$
\phi_{\pi p} \rightarrow \phi_{p}^{W W}, \quad \phi_{\pi \sigma} \rightarrow \phi_{\sigma}^{W W}, \quad \eta_{\sigma} \rightarrow 1 .
$$

In $[16,30,50,51]$ the Fock-Schwinger gauge has been used instead of the light-cone one. With the Fock-Schwinger gauge one obtains a recursion formula for the moments of the various twist-3 pion DAs from which one can also determine 2-particle twist-3 DAs for a given 3-particle DA. However, the 2-particle DAs determined from the recursion formula differ from ours for the same 3-particle DA markedly. If the Fock-Schwinger gauge is employed, the Wilson lines are not unity and are of significance. We expect that a consistent calculation of the subprocess amplitudes using either the light-cone gauge or the Fock-Schwinger one in the vacuum-particle matrix elements leads to the same results. At least for the case of electroproduction of a transversely polarized $\rho$-meson the equivalence of the two methods has been shown [24].

\section{APPENDIX B: 2-PARTICLE AND 3-PARTICLE TWIST-3 CONTRIBUTIONS TO SUBPROCESS AMPLITUDES}

In this section we list and comment on the separate 2- and 3-particle twist-3 contributions. Their sum is given in (33). As mentioned above, the 2-particle contribution can be completely expressed through the combination of 2-particle twist-3 DAs appearing on the left-hand side of the equations of motion (A11) and (A12) and can thus be simplified to

$$
\begin{aligned}
\mathcal{H}_{0-\lambda, \mu \lambda}^{\mathrm{twist}-3,2-\text { particle }}= & 4 \pi \alpha_{s}\left(\mu_{R}^{2}\right) f_{\pi} \mu_{\pi} \frac{C_{F}}{N_{C}} \frac{\sqrt{-\hat{u} \hat{s}}}{\sqrt{2} \hat{s}^{2} \hat{u}^{2}} \int_{0}^{1} d \tau \phi_{2}^{\mathrm{EOM}}(\tau) \\
& \times\left[\left(\frac{2 \lambda-\mu}{2(1-\tau)^{2}}+\frac{2 \lambda+\mu}{2 \tau(1-\tau)}\right)\left(\hat{s}^{2}+\hat{u}^{2}\right)\right. \\
& \left.+\mu \frac{\hat{t} \hat{s}}{\tau(1-\tau)}\right] .
\end{aligned}
$$

On the other hand, for the 3-particle twist-3 amplitude we derive 


$$
\begin{aligned}
\mathcal{H}_{0-\lambda, \mu \lambda}^{\text {twist } 3,3-\text { particle }}= & 4 \pi \alpha_{s}\left(\mu_{R}^{2}\right) \frac{f_{3 \pi}}{N_{C}} \frac{\sqrt{-\hat{u} \hat{s}}}{\sqrt{2} \hat{s}^{2} \hat{u}^{2}} \\
& \times \int_{0}^{1} d \tau \int_{0}^{1-\tau} \frac{d \tau_{g}}{\tau_{g}} \phi_{3 \pi}\left(\tau, 1-\tau-\tau_{g}, \tau_{g}\right) \\
& \times\left\{( 2 \lambda - \mu ) \left[C_{F}\left(\frac{1}{1-\tau}-\frac{1}{1-\tau-\tau_{g}}\right) \frac{\hat{s}^{2}+\hat{u}^{2}}{\tau_{g}}\right.\right. \\
& \left.+\left(C_{F}-\frac{C_{A}}{2}\right)\left(\frac{1}{\tau}+\frac{1}{1-\tau-\tau_{g}}\right) \frac{\hat{t}^{2}}{\tau_{g}}\right] \\
& \left.-\frac{2 \lambda+\mu}{\tau(1-\tau)}\left(\hat{s}^{2}+\hat{u}^{2}\right)-2 \mu \frac{\hat{s} \hat{t}}{\tau(1-\tau)}\right\} .
\end{aligned}
$$

The 2- and 3-particle twist-3 contributions do not respect current conservation separately; only their sum does so. However, the terms proportional to the color factor $C_{A}$ occurring only in (B2) are gauge invariant separately. Note that both (B1) and (B2) possess terms that are not symmetric in $\hat{s} \leftrightarrow \hat{u}$, i.e., do not obey the crossing properties expected for this process on general grounds [33]. However, their sum (33) is symmetric in $\hat{s} \leftrightarrow \hat{u}$; i.e., the expected crossing symmetry is recovered in the full twist-3 contribution.
[1] A. V. Radyushkin, Phys. Rev. D 58, 114008 (1998).

[2] M. Diehl, T. Feldmann, R. Jakob, and P. Kroll, Eur. Phys. J. C 8, 409 (1999).

[3] M. Diehl and P. Kroll, Eur. Phys. J. C 73, 2397 (2013).

[4] A. Danagoulian et al. (Hall A Collaboration), Phys. Rev. Lett. 98, 152001 (2007).

[5] H. W. Huang and P. Kroll, Eur. Phys. J. C 17, 423 (2000).

[6] H. W. Huang, R. Jakob, P. Kroll, and K. Passek-Kumerički, Eur. Phys. J. C 33, 91 (2004).

[7] P. Hoodbhoy and X. D. Ji, Phys. Rev. D 58, 054006 (1998).

[8] M. Diehl, Eur. Phys. J. C 19, 485 (2001).

[9] A. Airapetian et al. (HERMES Collaboration), Phys. Lett. B 682, 345 (2010).

[10] J. C. Collins, L. Frankfurt, and M. Strikman, Phys. Rev. D 56, 2982 (1997).

[11] S. V. Goloskokov and P. Kroll, Eur. Phys. J. C 65, 137 (2010).

[12] S. V. Goloskokov and P. Kroll, Eur. Phys. J. A 47, 112 (2011).

[13] G. R. Goldstein, J. O. Gonzalez Hernandez, and S. Liuti, Phys. Rev. D 91, 114013 (2015).

[14] M. Defurne et al. (Jefferson Lab Hall A Collaboration), Phys. Rev. Lett. 117, 262001 (2016).

[15] A. Sandacz (COMPASS Collaboration), J. Phys.: Conf. Ser. 938, 012015 (2017).

[16] V. M. Braun and I. E. Filyanov, Z. Phys. C 48, 239 (1990); Sov. J. Nucl. Phys. 52, 126 (1990); Yad. Fiz. 52, 199 (1990).

[17] M. Diehl, T. Feldmann, H. W. Huang, and P. Kroll, Phys. Rev. D 67, 037502 (2003).

[18] M. Diehl, T. Feldmann, R. Jakob, and P. Kroll, Eur. Phys. J. C 39, 1 (2005).

[19] T. Kitagaki et al., Phys. Rev. D 28, 436 (1983).

[20] P. Kroll, Eur. Phys. J. A 53, 130 (2017).

[21] S. Alekhin, J. Blümlein, and S. Moch, Phys. Rev. D 86, 054009 (2012).

[22] D. de Florian, R. Sassot, M. Stratmann, and W. Vogelsang, Phys. Rev. D 80, 034030 (2009).

[23] M. Göckeler, Ph. Hägler, R. Horsley, Y. Nakamura, D. Pleiter, P.E. L. Rakow, A. Schäfer, G. Schierholz,
H. Stüben, and J.M. Zanotti (QCDSF and UKQCD Collaborations), Phys. Rev. Lett. 98, 222001 (2007).

[24] I. V. Anikin, D. Y. Ivanov, B. Pire, L. Szymanowski, and S. Wallon, Nucl. Phys. B828, 1 (2010).

[25] I. V. Anikin and O. V. Teryaev, Phys. Lett. B 554, 51 (2003).

[26] M. Beneke and T. Feldmann, Nucl. Phys. B592, 3 (2001).

[27] P. Kroll and K. Passek-Kumerički, Phys. Rev. D 67, 054017 (2003).

[28] C. Patrignani et al. (Particle Data Group), Chin. Phys. C 40, 100001 (2016).

[29] T. Huang, X. H. Wu, and M.Z. Zhou, Phys. Rev. D 70, 014013 (2004).

[30] P. Ball, J. High Energy Phys. 01 (1999) 010.

[31] V. M. Braun, S. Collins, M. Gckeler, P. Prez-Rubio, A. Schäfer, R. W. Schiel, and A. Sternbeck, Phys. Rev. D 92 , 014504 (2015).

[32] J. B. Kogut and D. E. Soper, Phys. Rev. D 1, 2901 (1970).

[33] G. F. Chew, M. L. Goldberger, F. E. Low, and Y. Nambu, Phys. Rev. 106, 1345 (1957).

[34] M. C. Kunkel et al. (The CLAS Collaboration), arXiv: 1712.10314.

[35] V. L. Chernyak and A. R. Zhitnitsky, Phys. Rep. 112, 173 (1984).

[36] C. Shi, C. Chen, L. Chang, C. D. Roberts, S. M. Schmidt, and H. S. Zong, Phys. Rev. D 92, 014035 (2015).

[37] H. M. Choi and C.R. Ji, Phys. Rev. D 95, 056002 (2017).

[38] S. i. Nam and H. C. Kim, Phys. Rev. D 74, 096007 (2006).

[39] H. W. Huang, P. Kroll, and T. Morii, Eur. Phys. J. C 23, 301 (2002); 31, 279(E) (2003).

[40] H. Al Ghoul et al. (GlueX Collaboration), Phys. Rev. C 95, 042201 (2017).

[41] C. Fanelli et al., Phys. Rev. Lett. 115, 152001 (2015).

[42] D. J. Hamilton et al. (Jefferson Lab Hall A Collaboration), Phys. Rev. Lett. 94, 242001 (2005).

[43] B. Wojtsekhowski, in S. Ali et al., arXiv:1704.00816.

[44] T. Feldmann, P. Kroll, and B. Stech, Phys. Rev. D 58, 114006 (1998). 
[45] L. L. Frankfurt, P. V. Pobylitsa, M. V. Polyakov, and M. Strikman, Phys. Rev. D 60, 014010 (1999).

[46] P. Ball, V. M. Braun, and A. Lenz, J. High Energy Phys. 05 (2006) 004.

[47] A. V. Belitsky, D. Mueller, A. Kirchner, and A. Schafer, Phys. Rev. D 64, 116002 (2001).

[48] P. Kroll and A. Schäfer, Eur. Phys. J. A 26, 89 (2005).
[49] T. A. Armstrong et al. (Fermilab E760 Collaboration), Phys. Rev. D 56, 2509 (1997).

[50] A. Khodjamirian, T. Mannel, and P. Urban, Phys. Rev. D 67, 054027 (2003).

[51] A. S. Gorsky, Report No. ITEP-87-85, https://inspirehep .net/record/248789. 